\title{
Die Weichbodenfauna vor der Elbemündung unter dem Einfluß der Klärschlammverklappung
}

\author{
I. Die saisonale Veränderung von Arten- und Individuenzahl sowie Biomasse \\ unter Berücksichtigung der Sedimentstruktur \\ Ute Mühlenhardt-Siegel
}

Zoologisches Institut und Museum;

Martin-Luther-King-Platz 3, D-2000 Hamburg 13, Bundesrepublik Deutschland

\begin{abstract}
The soft bottom fauna near the River Elbe Estuary under the influence of sewage sludge. I. Seasonal variation of species number, abundance and biomass in relation to sediment structure. Macrobenthic fauna and sediment structure were studied at 6 stations from June 1979 to April 1981. The investigations were carried out in the outer estuary of the River Elbe at depths of 15-38 m near a sewage sludge dumping site. The sediment near this area consisted of a high portion of silt. Usually, very few species and small numbers of individuals were found near that dumping ground. During summer 1980, a mass development of Abra alba and several polychaete species caused an increase in species number which broke down during the following winter. The mass development observed in the study area was apparently not directly caused by the sludge dumping itself, but reflects the natural variability of the community structure. The number of species recorded was significantly correlated with a grain size fraction $>31 \mu \mathrm{m}$, especially in summer. The seasonal and spatial fluctuation of the benthic community could be well documented by the abundance per species (N/S index). The Shannon-Wiener-Index $\left(\mathrm{H}^{*}\right)$ and eveness $(\mathrm{J})$ were also useful indicators of community variability. The log-normal distribution did not show the seasonal and the spatial differences of the stations. Possible reasons are discussed. The biomass at the stations near the dumping area was less except during the mass development of $A$. alba. The average individual weight $(B / N)$ was shown to be a good indicator of population fluctuations; polychaete biomass was positively correlated to a grain size fraction $\leqq 6 \mu \mathrm{m}$, that of molluscs to a grain size fraction $>31 \mu \mathrm{m}$.
\end{abstract}

\section{EINLEITUNG}

Von 1962 bis Juni 1980 wurden im Ostteil der Deutschen Bucht über einem Areal von $3,5 \mathrm{~km}^{2}$ durchschnittlich $250000-300000 \mathrm{~m}^{3} \mathrm{Klärschlamm}$ pro Jahr aus der Hamburger Großkläranlage Köhlbrandhöft verklappt. Der Faulschlamm durfte nach einer Verweildauer von 20 Tagen in den Faultürmen der Kläranlage in die See eingebracht werden. Die Verklappung erfolgte in der Regel alle 2 Tage. Laut Verordnung des Deutschen Hydrographischen Institutes (Hamburg) sollten nicht mehr als $1260 \mathrm{~m}^{3}$ Abfallstoffe mit einem Festkörpergehalt von 5-7\% bei fahrendem Schiff in mindestens $2 \mathrm{~m}$ Wassertiefe und einer Fahrtgeschwindigkeit von möglichst $7 \mathrm{sm} / \mathrm{h}$ bei ablaufendem Wasser eingebracht werden.

Das Gebiet der Verklappung liegt in einem natürlichen Schlickgebiet der südöstlichen Deutschen Bucht. Gadow \& Schäfer (1973) führten die feinsten Partikel des 
Sediments im tiefen Wasser auf Antransport der Flüsse und Aufarbeitung älterer mariner Tone zurück. Der bemerkenswert hohe Anteil von Schluff $(<63 \mu \mathrm{m})$ in flachen Gebieten ist vermutlich auf spezielle hydrographische Bedingungen zurückzuführen (Figge, 1981). Creutzberg \& Postma (1979) korrelierten die Verteilung der siltigen Sedimente mit der Verteilung der maximalen Oberflächenströmung von $<1,67 \mathrm{~km} / \mathrm{h}$.

Schon oft wurde auf die enge Beziehung zwischen Korngröße und Besiedlung durch Makrofauna hingewiesen. Rhoads (1974) gab erfolgreiche Ansiedlungen bestimmter Nahrungserwerbstypen besonders auf weichen Böden an. Eisma (1966) beobachtete unterschiedliche Präferenzen einzelner Mollusca-Arten für Schlick- bzw. Sandsedimente. Auch Gray (1974) hielt die Korngröße für den bestimmenden Faktor in der Substratselektion. Neben der Korngrößenverteilung wird auch die Stabilität des Sediments als wichtig für die Struktur der Makrofaunabesiedlung erachtet (Young \& Rhoads, 1971). Veränderungen am Beispiel der Polychaetenfauna in Monterey Bay (Kalifornien, USA) wurden nach Hulberg \& Oliver (1980) an Hand von Käfigversuchen auf Sedimenthabitat-Veränderungen zurückgeführt.

Auch die Wirkung von Streß - im Sinne von Franz (1981) oder Gray (1982) - auf die Bodentiere wurde oft beobachtet. Sowohl Gifte wie Schwermetalle und chlorierte Kohlenwasserstoffe im Faulschlamm als auch Abwässer können offensichtlich nekrotische Läsionen bei Krebsen hervorrufen (Dybern, 1972; Young \& Pearce, 1975). Als besonders sensitiv gegen Verschmutzung gelten Amphipoda (Pearce, 1972; Parker, 1980), die in belasteten Gebieten fehlen (Botton, 1979). Auch von Schalendeformationen bei Macoma baltica durch Abwassereinfluß wurde berichtet (Rosenberg et al., 1975). Meist war eine Reduzierung der Artenzahl in den belasteten Gebieten zu beobachten (Tulkki, 1969; Young \& Pearce, 1975; McIntyre, 1978; Soulsby et al., 1978; Botton, 1979; Parker, 1980; Pearson, 1980; Rachor, 1980). Von hoher Biomasse bei gleichzeitig stark erniedrigter Artenzahl in Gebieten mit hoher Belastung durch organische Substanz berichteten Topping \& McIntyre (1972), Stirn (1973), Caspers (1979), Gray (1979), Rapport et al. (1981), Probert (1981), Pearson (1981), Gray (1982) und Eleftheriou et al. (1982). Dagegen konnten Jenkinson (1972) für das Seegebiet um die Isle of Wight (Südengland) und Moore (1979) für den Firth of Forth (Schottland) keine Veränderungen der Bodenfauna in klärschlammbelasteten Gebieten nachweisen. Tulkki (1969) hielt die Zusammensetzung der Bodentiergemeinschaften, Individuendichte und Biomasse der Tiere für gute Kriterien zur Bewertung des Naturzustandes und des Verunreinigungsgrades, die mit hydrographischen Faktoren zusammenhängen.

Ein Ziel der vorliegenden Arbeit war die quantitative Erfassung der Zusammenhänge zwischen Bodenfauna und Sedimenteigenschaften im genannten Gebiet. Als Parameter wurden zunächst Korngröße und Wassergehalt des Sediments untersucht. Weiterhin sollte geprüft werden, ob der Klärschlamm als Streßfaktor für die Bodentiergemeinschaft anzusehen ist. Hierzu wurden Artenzahl, Individuenabundanz und Biomasse der Makrofauna bestimmt.

Außerdem wurden für die Interpretation hilfreiche Diversitätsindices errechnet, welche die Verteilung der Individuen auf die Arten beschreiben. Saisonale Schwankungen spielen bei den gesamten Parametern eine große Rolle; sie wurden bei der vorliegenden Analyse berücksichtigt. 


\section{MATERIAL UND METHODE}

Für die vorliegende Arbeit wurden von Juni, 1979 bis April 1981 regelmäßig Bodenproben mit einem van Veen Backengreifer der Fläche $0,1 \mathrm{~m}^{2}$ an 5 Dauerstationen (Abb. 1) genommen. Die Probennahme fand in folgenden Monaten statt: 6/79, 7/79, 10/79,

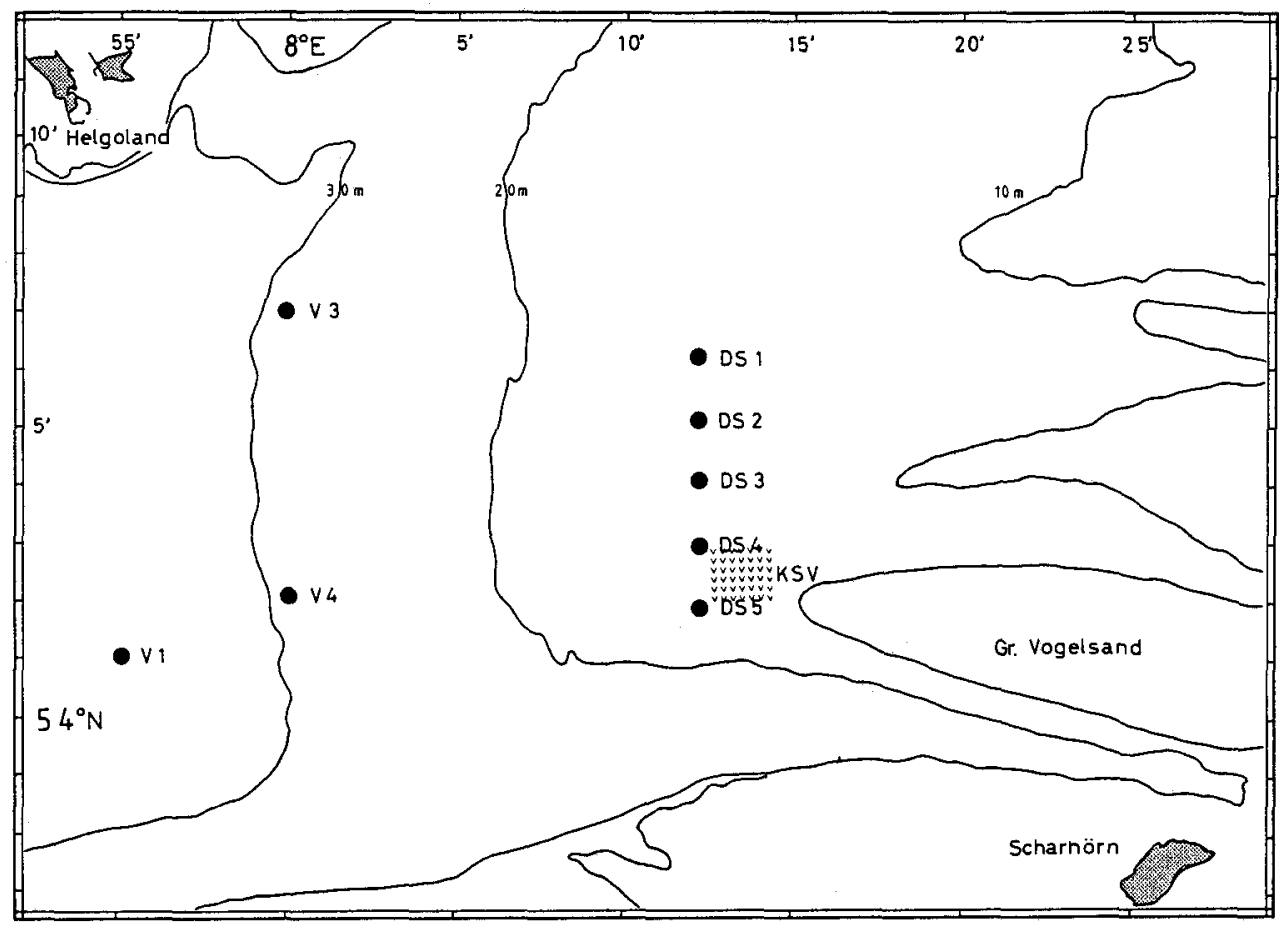

Abb. 1. Untersuchungsgebiet in der Deutschen Bucht südöstlich von Helgoland. DS 1 - DS 5 kennzeichnet die Dauerstationen, $\mathrm{V}=$ Vergleichsstationen, $\mathrm{KSV}=$ Klärschlammverklappungsgebiet ( $\mathrm{V} 2$ liegt nördlich von Helgoland)

$11 / 79,3 / 80,4 / 80,6 / 80,7 / 80,2 / 81$ und 4/81. Sie erfolgte mit den FSen "Friedrich Heincke" (11/79), "Gauss" (7/80, 4/81) und "Victor Hensen" (übrige Probendaten).

Die Positionen und ihre Wassertiefen sind in Tabelle 1 angegeben

Tabelle 1. Positionsangaben

\begin{tabular}{|ccc|}
\hline Station & Position & $\begin{array}{c}\text { Wasser- } \\
\text { tiefe (m) }\end{array}$ \\
\hline DS 1 & $54^{\circ} 06,2^{\prime} \mathrm{N} / 08^{\circ} 12,2^{\prime} \mathrm{E}$ & 18 \\
DS 2 & $54^{\circ} 05,2^{\prime} \mathrm{N} / 08^{\circ} 12,2^{\prime} \mathrm{E}$ & 17 \\
DS 3 & $54^{\circ} 04,1^{\prime} \mathrm{N} / 08^{\circ} 12,2^{\prime} \mathrm{E}$ & 15 \\
DS 4 & $54^{\circ} 03,0^{\prime} \mathrm{N} / 08^{\circ} 12,3^{\prime} \mathrm{E}$ & 15 \\
DS 5 & $54^{\circ} 01,9^{\prime} \mathrm{N} / 08^{\circ} 12,3^{\prime} \mathrm{E}$ & 16 \\
\hline
\end{tabular}


Zusätzlich dazu wurden jeweils an einer "Vergleichs-Station", die auf Grund ihrer Sedimentbeschaffenheit den Dauerstationen ähnelte, Proben entnommen. Leider waren nur in größeren Wassertiefen Stationen mit Weichböden zum Vergleich heranzuziehen. Die Positionen und ihre Tiefen waren:

Tabelle 2. Positionsabgaben von Vergleichsstationen

\begin{tabular}{|cllcc|}
\hline Station & \multicolumn{2}{c}{ Zeitraum } & Position & Tiefe m \\
\hline V 1 & Oktober & 1979 & $54^{\circ} 01,7^{\prime} \mathrm{N} / 07^{\circ} 55,0^{\prime} \mathrm{E}$ & 34 \\
V 2 & November 1979 & $54^{\circ} 13,4^{\prime} \mathrm{N} / 07^{\circ} 45,0^{\prime} \mathrm{E}$ & 38 \\
V 3 & März & 1980 & $54^{\circ} 07,0^{\prime} \mathrm{N} / 07^{\circ} 59,8^{\prime} \mathrm{E}$ & 30 \\
& April & 1980 & & \\
V 4 & Juni & 1980 & $54^{\circ} 02,3^{\prime} \mathrm{N} / 07^{\circ} 59,9^{\prime} \mathrm{E}$ & 28 \\
& Februar & 1981 & & \\
\hline
\end{tabular}

Pro Station wurden 5 Parallelproben genommen, was nach Lie (1968) zur adäquaten Erfassung der biologischen Daten und ihrer statistischen Auswertung ausreicht. Diesen Proben wurde eine Sedimentprobe für Sedimentanalysen entnommen und eingefroren. Der restliche Greiferinhalt wurde über einem Sieb der Maschenweite 0,5 mm gespült und mit gepuffertem $5 \%$ igem Formaldehyd fixiert.

An den gefrosteten Sedimentproben wurden Wassergehalts- und Korngrößenanalysen durchgeführt. Zur Bestimmung der Korngröße wurde die Pipettanalyse verwendet, ein Sedimentationsverfahren, das besonders für kleine Korngrößen von $<31 \mu \mathrm{m}$ geeignet ist (Müller, 1964).

Ferner wurden die wöchentlichen Meßdaten vom Feuerschiff "Elbe 1" für Salinität und Temperatur vom Sommer 1978 bis Sommer 1981 verwendet (Deutsches Hydrographisches Institut, 1979 bis 1982).

Das fixierte Tiermaterial der quantitativen Bodenproben wurde bis zur Art bestimmt und ausgezählt. In Ausnahmefällen war bei einigen Juvenilen nur eine Bestimmung bis zur Gattung möglich. Die Angabe der Biomasse erfolgte in Gramm aschefreies Trockengewicht (AFTG), nach einer Trocknung bei $70^{\circ} \mathrm{C}$ und Glühen bei $510^{\circ} \mathrm{C}$ (MühlenhardtSiegel, 1981).

\section{STATISTISCHE AUSWERTUNG}

Nach Gray (1981) ist die log-Normalverteilung eine gute Beschreibung für die Verteilung der Individuen auf die Arten in einer Benthosgemeinschaft. Für die Dauerstationen wurde beispielhaft für jede Jahreszeit die Individuenhäufigkeitsverteilung pro Art auf die log-Normalverteilung geprüft. Hierzu wurden die Parallelproben je einer Station zusammengefaßt.

Aus Arten- und Individuendichte wurden für jede Probe der Shannon-Wiener-Index $\mathrm{H}^{\prime}$ und die "eveness" J errechnet, wobei zur Berechung von $\mathrm{H}^{\prime}$ der natürliche Logarithmus verwandt wurde (Pielou, 1976):

$$
H^{\prime}=\sum_{i=1}^{s} p_{i} \cdot \ln \cdot p_{i}, \quad p_{i}=\frac{n_{i}}{N}, J=\frac{H^{\prime}}{H_{\text {max }}^{\prime}}
$$


$\mathrm{n}_{\mathrm{i}}=$ Individuendichte der $\mathrm{i}$-ten Art, $\mathrm{N}=$ Gesamtindividuenzahl, $\mathrm{S}=$ Artenzahl, $\mathrm{H}_{\max }^{\prime}=$ In $\mathrm{S}$.

Zwischen den abiotischen und biotischen Parametern jeder Probe wurden nichtlineare bzw. lineare Regressionen berechnet und mit Hilfe des F-Tests (Sachs, 1984) auf ihre Signifikanz geprüft. Als signifikant gewertet wurden Korrelationen mit einem Signifikanzniveau unter $5 \%$. Wurde eine abhängige Variable von mehreren unabhängigen Variablen beeinflußt, konnte mit Hilfe multilinearer Regressionen und der Varianzen ein Hinweis darauf gefunden werden, welche unabhängige Variable den stärksten Einfluß besaß.

\section{ERGEBNISSE}

\section{Saisonalität}

\section{Abiotische Faktoren}

Die mittlere Korngrößenzusammensetzung (Abb. 2) zeichnet sich dadurch aus, daß hohe Anteile den groben Fraktionen $(>31 \mu \mathrm{m})$ und den feinsten Fraktionen mit Korngrößen unter $8 \mu \mathrm{m}$ zukommen. Allen Stationen gemeinsam ist ein niedriger Anteil der feinen Fraktionen (unter $31 \mu \mathrm{m}$ ) im Frühjahr. Die höchsten Werte im Untersuchungszeitraum waren an den Stationen 1, 2 und $3 \mathrm{im}$ Juli 1980 zu finden, an Station 4 im Februar 1981. Einen von den nördlichen Stationen unterschiedlichen Jahresgang zeigt die Korngrößenverteilung an der Station 5, obwohl auch hier der höchste Anteil der feinen Korngrößen im Februar 1981 zu finden war.

Nur geringe Schwankungen im Jahresgang zeigen die Vergleichsstationen (Abb. 2). Der höchste Anteil der kleinen Korngrößen war im April'1980 zu finden.

Aus den Temperaturmessungen von Feuerschiff "Elbe 1" (DHI, 1979-1982) geht hervor, daß im Untersuchungszeitraum die Bodentemperatur von Februar bis September unter dem langjährigen Monatsmittel (Goedecke, 1954), von September bis Dezember/ Januar aber über dem langjährigen Monatsmittelwert lag (Abb. 3). Hierbei wird auch der kalte Winter 1978/79 deutlich.

\section{Arten- und Individuenzahl}

Die maximal an einer Station gefundene Artenzahl S zeigte im Untersuchungszeitraum einen jahreszeitlich bedingten Verlauf, der allen Dauerstationen gemeinsam war. Er hatte eine zunehmende Tendenz zum Sommer (Juli) und nahm zum Herbst hin wieder ab. Im Frühjahr 1980 wurde dieser Verlauf durch einen kurzfristigen Artenanstieg im März unterbrochen (Abb. 4). Neben den Massenentwicklungen einzelner Arten hatten im Juli 1980 alle Stationen einen bedeutenden Artenzuwachs, der am höchsten an der nördlichen Station 1 und am niedrigsten an Station 2 war (Mühlenhardt-Siegel, 1985).

Die über den gesamten Untersuchungszeitraum gemittelte Artenzahl zeigte den höchsten Wert an Station 1 mit 27,4 Arten. Nach Süden bis zur Station 4 (16,2 Arten) war ein stetiger Artenverlust zu bemerken. Die südlichste Dauerstation 5 hatte den zweithöchsten Wert $(21,4)$. Die Streuung dieser gemittelten Artenzahlen lag mit Werten von 5 bis 7 relativ hoch, was durch die sehr hohen Artenzahlen an allen Dauerstationen im Juli 1980 bedingt war. Dabei war die Schwankung in der Artenzahl vor allem an den Stationen 3 und 4 in Nähe des Einbringungsgebietes besonders hoch. 

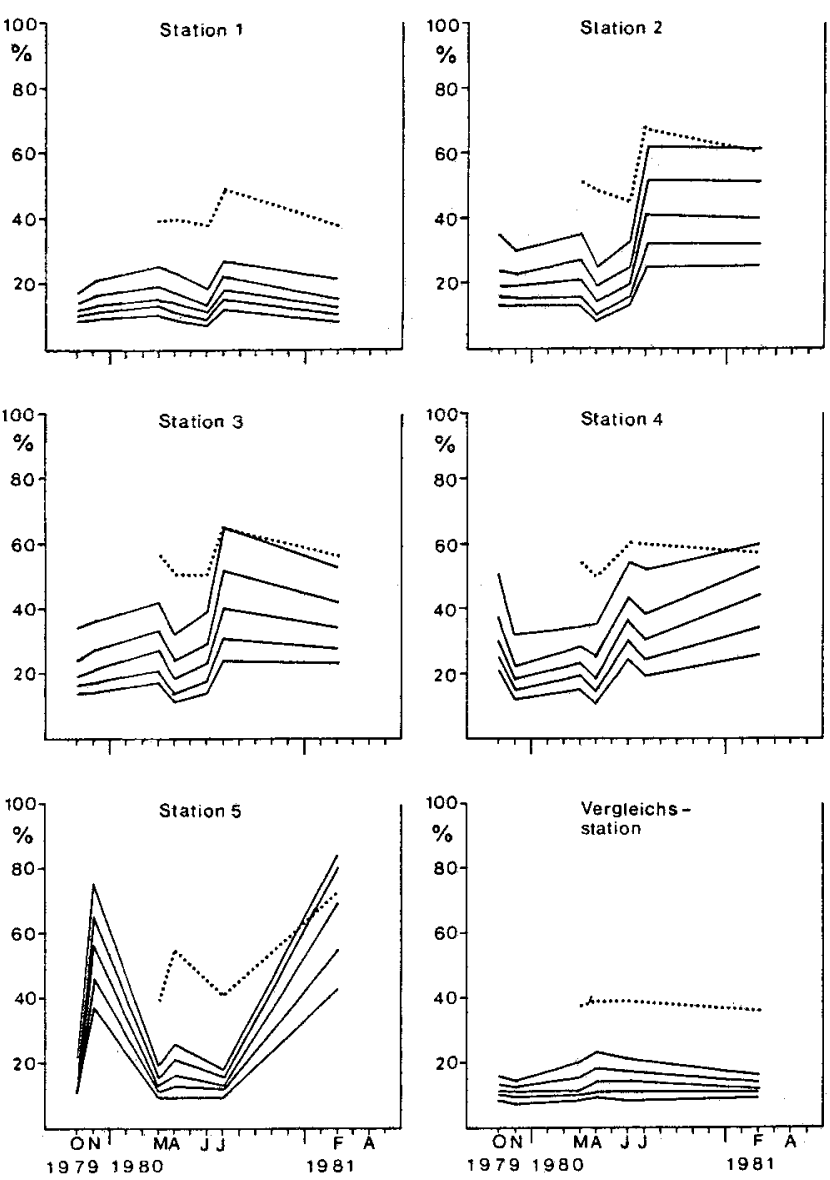

Abb. 2. Korngrößenzusammensetzung des Sediments der Dauerstationen und dessen Wassergehalt (gepunktete Linie). Die durchgezogenen Linien geben folgende Korngrößenfraktionen in Prozent der Gesamtfraktion an (von oben nach unten): $\leqq 31 \mu \mathrm{m}, \leqq 16 \mu \mathrm{m}, \leqq 8 \mu \mathrm{m}, \leqq 4 \mu \mathrm{m}, \leqq 2 \mu \mathrm{m}$

Die Vergleichsstationen zeigten einen jahreszeitlich bedingten Verlauf der Artenzahl, der dem der Dauerstationen entgegenlief. Die Artenzahlen lagen mit 40 bis 60 Arten hier stets höher als an den Dauerstationen.

Insgesamt wurden im Untersuchungsgebiet 120 Arten gefunden. Davon waren 46 Polychaeta, 36 Crustacea, 23 Mollusca, 5 Echinodermata und 10 "Sonstige". Tabelle 1 gibt Auskunft über das Auftreten der einzelnen Arten an den Dauer- und Vergleichsstationen.

Die dominanten Arten Nephtys hombergi, Pectinaria koreni, Abra alba, Nucula nitida, Diastylis rathkei und Ophiura texturata hatten eine Stetigkeit von mehr als $90 \%$ an den Dauerstationen im Untersuchungszeitraum. Hingegen traten 30 der 120 Arten im Untersuchungsgebiet nur an den Vergleichsstationen auf.

Auch die mittlere Individuenzahl ließ an den Dauerstationen einen jahreszeitlichen Trend erkennen (Abb. 5). Hier zeigt sich eine Abnahme der Individuenzahl vom Früh- 


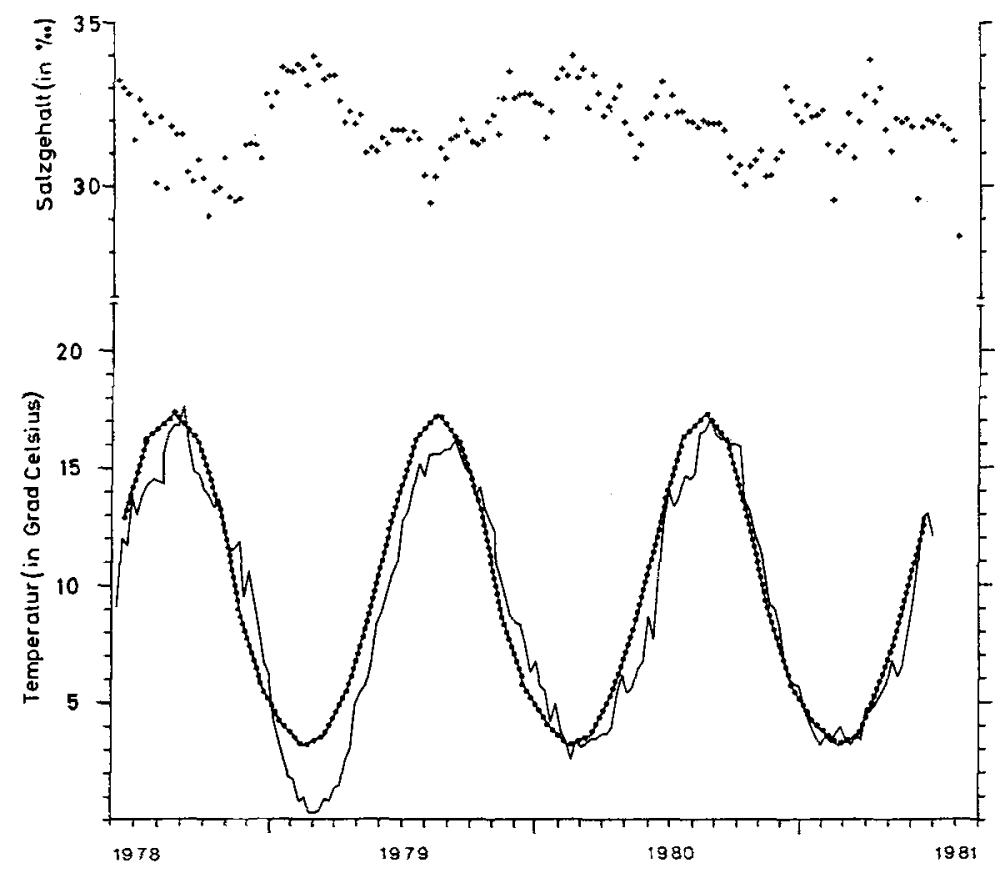

Abb. 3. Temperatur und Salzgehalt am Boden in den Jahren 1978 bis 1981. Daten der Messungen von Feuerschiff "Elbe 1" (gepunktete Linie das langjährige Monatsmittel)

Arten-

zahl
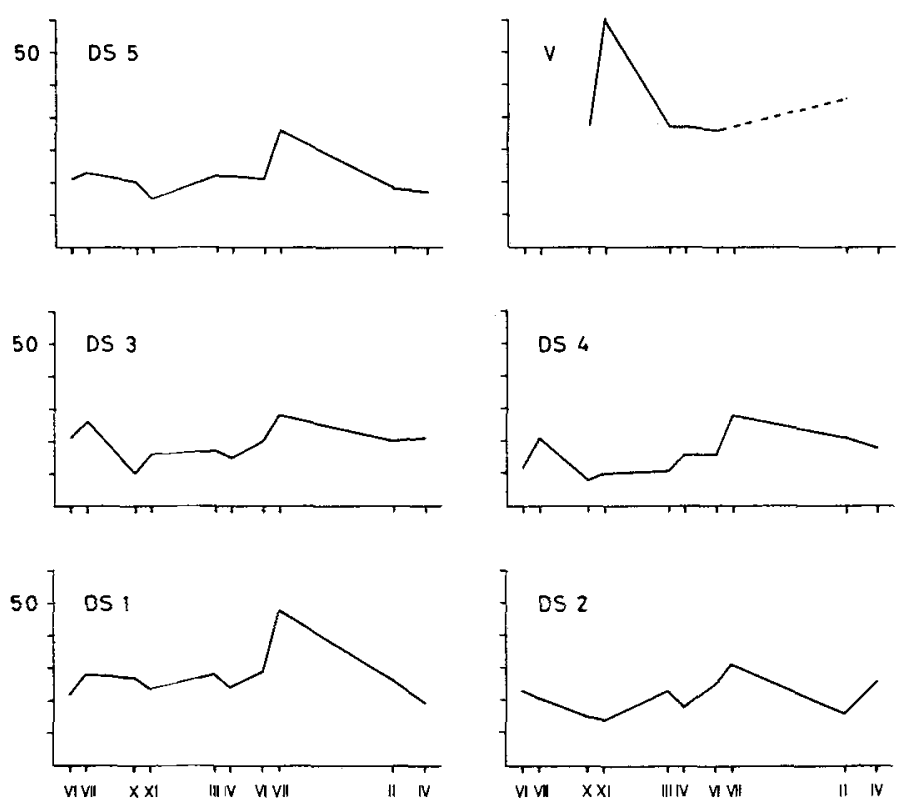

Abb. 4. Veränderung der Artenzahl im Jahresgang an den Dauerstationen 1 bis 5 und an den Vergleichsstationen V (römische Ziffern bezeichnen die Monate). Gepunktete Linie: für den Zeitraum liegen keine kontinuierlichen Messungen vor 

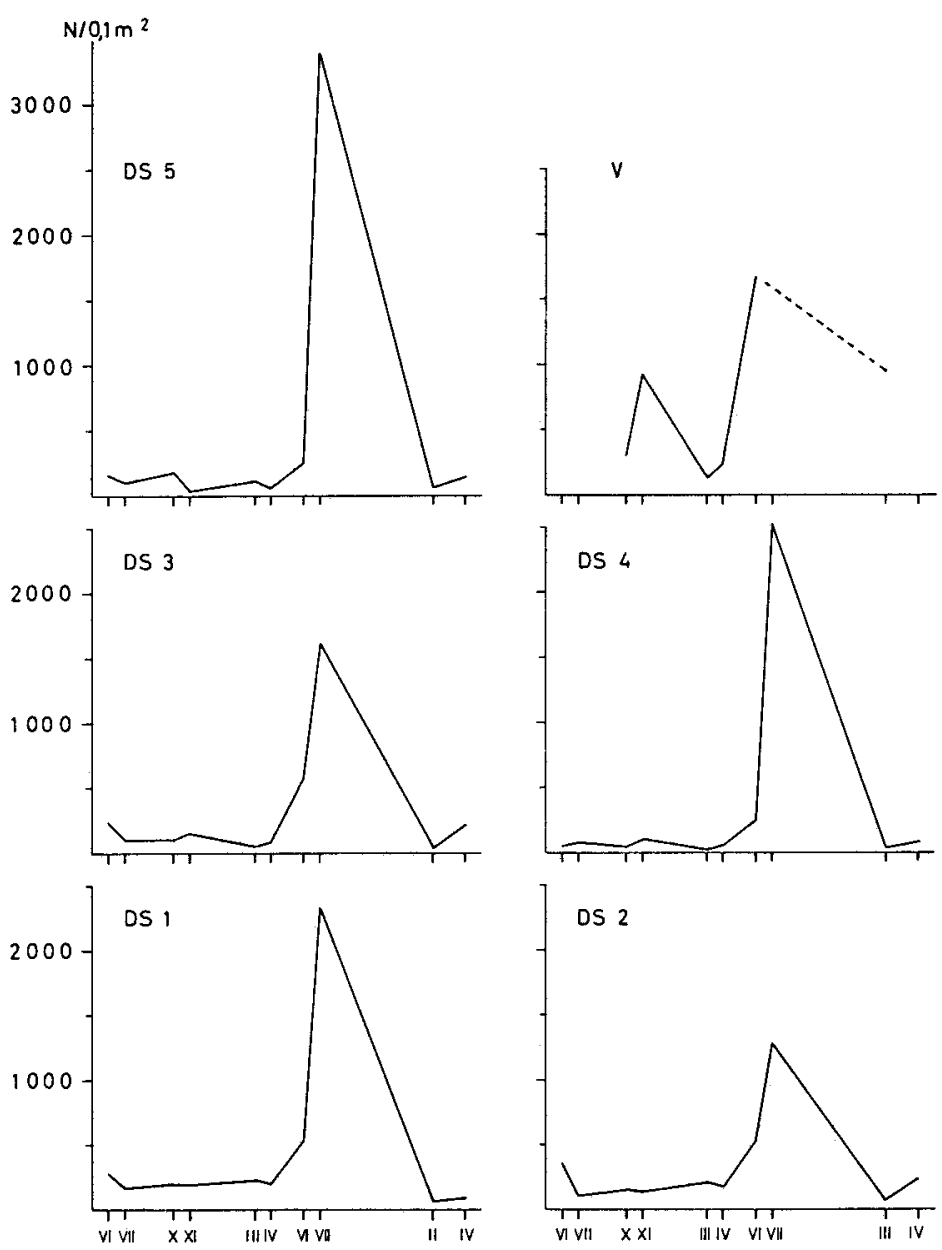

DS 2

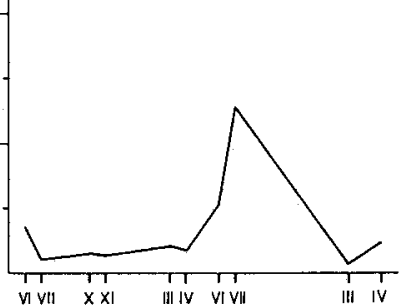

Abb. 5. Veränderung der Gesamtindividuenzahl (N/0,1 $\mathrm{m}^{2}$ ) an den Dauerstationen 1 bis 5 und den Vergleichsstationen V (römische Ziffern bezeichnen die Monate). Gepunktete Linie: für den Zeitraum liegen keine kontinuierlichen Messungen vor

sommer zum Hochsommer 1979, gefolgt von einer stetigen Zunahme, die bis März 1980 anhielt. Diese Individuenzunahme ist auf eine Ansiedlung juveniler Diastylis rathkei und Ophiura texturata zurückzuführen. Vom März bis April 1980 erfolgte ein Rückgang in der Abundanz, der mit dem Absterben der Adulten des Vorjahres zu begründen ist (z. B. Pectinaria koreni, Nucula nitida). Zum Juni bis einschließlich Juli 1980 war durch eine Massenentwicklung von Abra alba und einiger Polychaeten-Arten die Individuendichte sehr stark angestiegen, die im Winter (Februar 1981) wieder geringer war. Im April 1981 war ein erneuter Anstieg der Individuenzahl zu bemerken, diesmal ausschließlich verursacht durch juvenile Diastylis rathkei.

Ein Überblick über die häufigsten Arten an den Dauerstationen zeigt, daß neben den sechs zuvor genannten Hauptarten die Polychaeta Scalibregma inflatum, Scoloplos 
armiger, Anaitides sp. und Pholve minuta sowie die Bivalve Macoma baltica häufig vertreten waren (Mühlenhardt-Siegel, 1985).

Vergleicht man die Individuenzahlen an den Stationen im Mittel über den Untersuchungszeitraum, zeigt sich ein hoher Wert für Station 1 und eine minimale Abundanz an Station 4. Bedingt durch die Massenentwicklung im Sommer 1980 ist die Streuung sehr hoch. Die Massenentwicklung wird zuerst im Juni 1980 an Station 5 deutlich und hatte sich im Juli nach Norden hin bis Station 2 ausgedehnt.

\section{Biomasse}

Im Vergleich der Dauerstationen zeichnet sich ein allen Stationen gemeinsamer Trend $a b$, wonach im Sommer die höchsten und im Winter die niedrigsten Biomassewerte anzutreffen waren (Abb. 6). Im Mittel lag die höchste Biomasse an der südlichsten

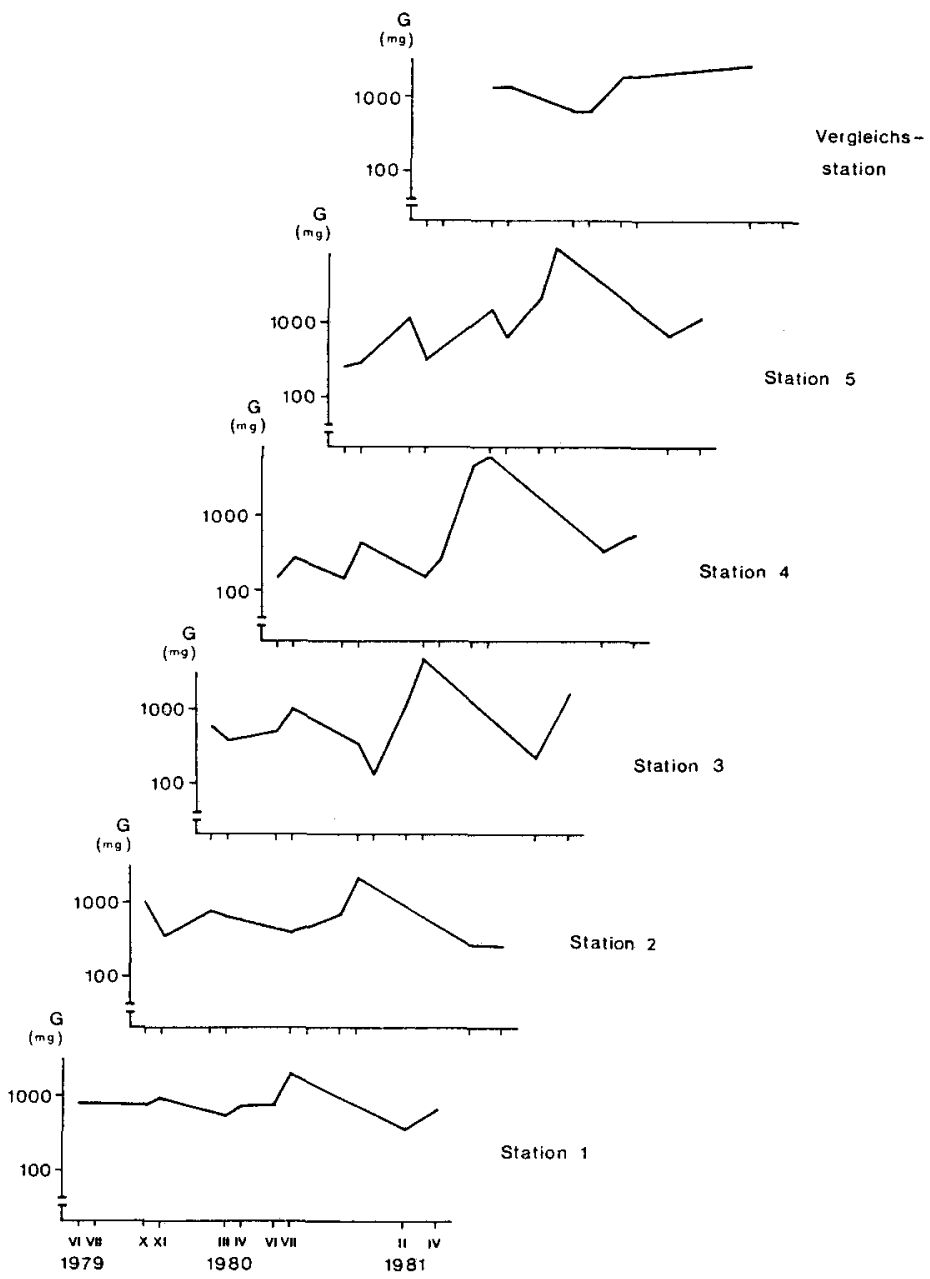

Abb. 6. Jahresgang der Biomasse $\mathrm{G}$ (aschefreies Trockengewicht in $\mathrm{mg} / 0,1 \mathrm{~m}^{2}$ ) an den Dauerstationen 1 bis 5 und den Vergleichsstationen $\mathrm{V}$ 
Station 5 mit 17,594 g AFTG/m² vor, während die nach Norden anschließenden Stationen 4 und 3 Werte um $10 \mathrm{~g} \mathrm{AFTG} / \mathrm{m}^{2}$ erreichten. Die beiden nördlichsten Stationen 1 und 2 lagen mit Mengen von 8,089 g AFTG/m $\mathrm{m}^{2}$ (Station 1) und 7,213 g AFTG/m ${ }^{2}$ (Station 2) weit darunter. Da hohe Gewichte an den drei südlichen Stationen nicht regelmäßig auftraten, wurden die höchsten Werte im Untersuchungszeitraum für eine weitergehende Beurteilung weggelassen.

Ohne Berücksichtigung der Abra alba-Massenentwicklung wiesen mit Ausnahme der Station 4 alle Stationen annähernd gleiche Werte auf $\left(5,50\right.$ bis $\left.8,80 \mathrm{~g} \mathrm{AFTG} / \mathrm{m}^{2}\right)$. Allerdings traten im Jahresgang nur an den beiden nördlichen Stationen geringe Schwankungen auf. Die Variabilität der Biomasse zwischen den Parallelproben war im Frühsommer und Herbst niedrig, im Sommer und Winter hingegen hoch (Tab. 3). Das dominierende Taxon bezüglich der Biomasse waren die Mollusca (Abb. 7), hauptsächlich repräsentiert durch Nucula nitida. Eine Ausnahme bildete Station 4, wo Abra alba die Hauptart war. Neben dieser Art waren hier im Jahr 1979 noch Macoma baltica und Ensis directus (Cosel et al., 1982; Mühlenhardt-Siegel et al., 1983) dominante Arten unter den Mollusca.

Die zweitwichtigste Gruppe in der Biomassezusammensetzung waren die Polychaeta. 1979 und 1980 wurden sie meist von der Art Pectinaria koreni repräsentiert, der Anteil von Nephtys hombergi nahm aber im Verlauf des Jahres 1980 zu und war 1981 höher als der von Pectinaria koreni. 1980 war der Anteil dieser beiden Polychaeta-Arten vergleichsweise gering, bedingt durch die schon oben erwähnte Massenentwicklung anderer Polychaeta-Arten.

Die Echinodermata hatten im Gegensatz zur Individuendominanz oft einen hohen Anteil an der Gewichtsdominanz. Sie folgten vor allem an den südlichen Stationen den Mollusca und waren überwiegend durch Ophiura texturata vertreten. Die Crustacea stellten nur einen geringen Anteil der Gesamtbiomasse.

An den Vergleichsstationen dominierte unter den Mollusca stets Nucula nitida, unter den Polychaeta Nephtys hombergi und bei den Echinodermata die Art Ophiura albida.

\section{Beziehung der Sedimentfaktoren zur Artenzahl und Biomasse}

Die Beziehung zwischen den Sedimenteigenschaften Korngröße und Wassergehalt und der Artenzahl an den Dauerstationen wurde mittels Regressionsberechnungen analysiert und in Tabelle 4 dargestellt.

Die Gesamtartenzahl war positiv mit den gröberen Korngrößenanteilen über $31 \mu \mathrm{m}$ korreliert, entsprechend negativ mit allen kleineren Sedimentfraktionen. Die Beziehung zwischen Wassergehalt und Gesamtartenzahl war ebenfalls negativ. Allein die Artenzahl der Polychaeta und Mollusca zeigten unter den Hauptgruppen Korrelationen zu den Sedimenteigenschaften. Diese Beziehungen waren ebenfalls negativ.

Die Höhe des Korrelationskoeffizienten $\mathrm{r}$ zeigte eine Beziehung zur Jahreszeit insofern, als sie in Herbst und Frühjahr gering war; im April bestand keine Beziehung zwischen Artenzahl und Sediment, im Sommer hingegen war die Korrelation zwischen Artenzahl und Sedimenteigenschaften sehr hoch. Dieser Jahresgang in der Beziehung ist vermutlich folgendermaßen zu erklären: Durch häufigere Stürme in Spätherbst und Winter sind die Sedimente gut umgelagert und durchmischt. Auf diesem Substrat siedeln im Frühjahr viele Jungtiere diverser Arten. Im Sommer, durch überwiegend ruhige 


\begin{tabular}{|c|c|}
\hline & 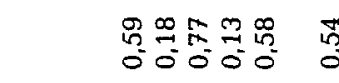 \\
\hline 苟 & 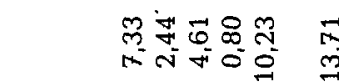 \\
\hline$\sum^{5}$ & 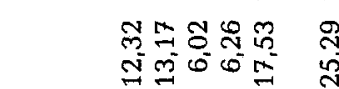 \\
\hline$>$ & 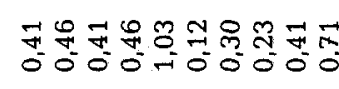 \\
\hline is in & 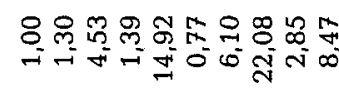 \\
\hline 10 & 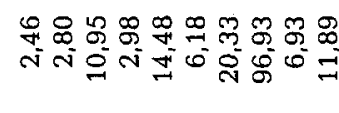 \\
\hline$>$ & $\begin{array}{l}M \\
3 \\
0 \\
0\end{array}$ \\
\hline$\nabla i$ & \begin{tabular}{l}
9 \\
\hdashline \\
0 \\
0 \\
0
\end{tabular} \\
\hline 10 & 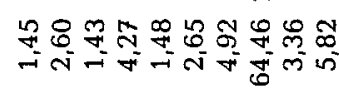 \\
\hline$>$ & 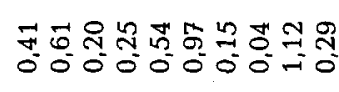 \\
\hline$n$ in & 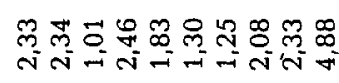 \\
\hline $\mid m$ & 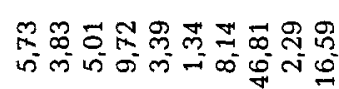 \\
\hline$>$ & 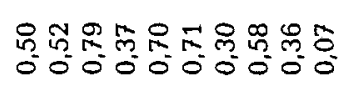 \\
\hline$N \infty$ & 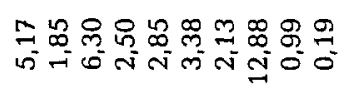 \\
\hline 10 & 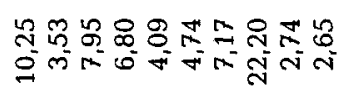 \\
\hline$>$ & 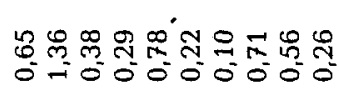 \\
\hline$-\infty$ & 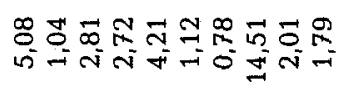 \\
\hline 10 & 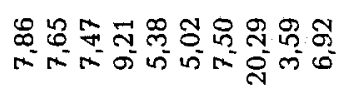 \\
\hline 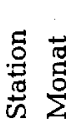 & 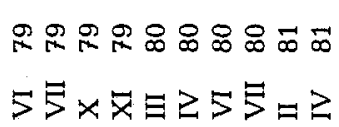 \\
\hline
\end{tabular}



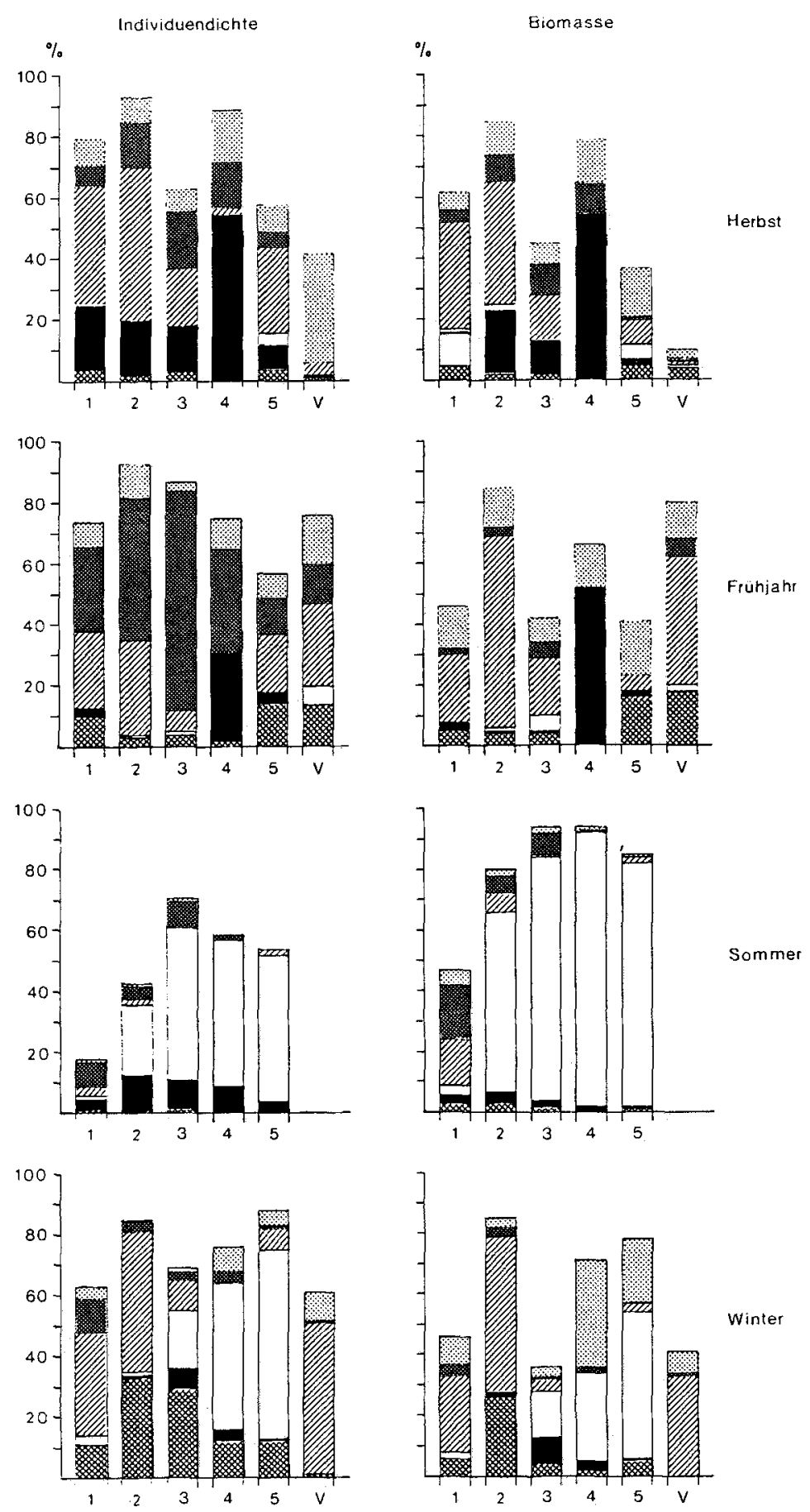

o. texturata

D. rathkei

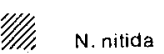

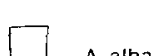

P. koreni

N. nombergi

Abb. 7. Individuen- und Biomassendominanz der häufigsten Arten an den Dauerstationen 1 bis 5 und den Vergleichsstationen $\mathrm{V}$ zu den verschiedenen Jahreszeiten 


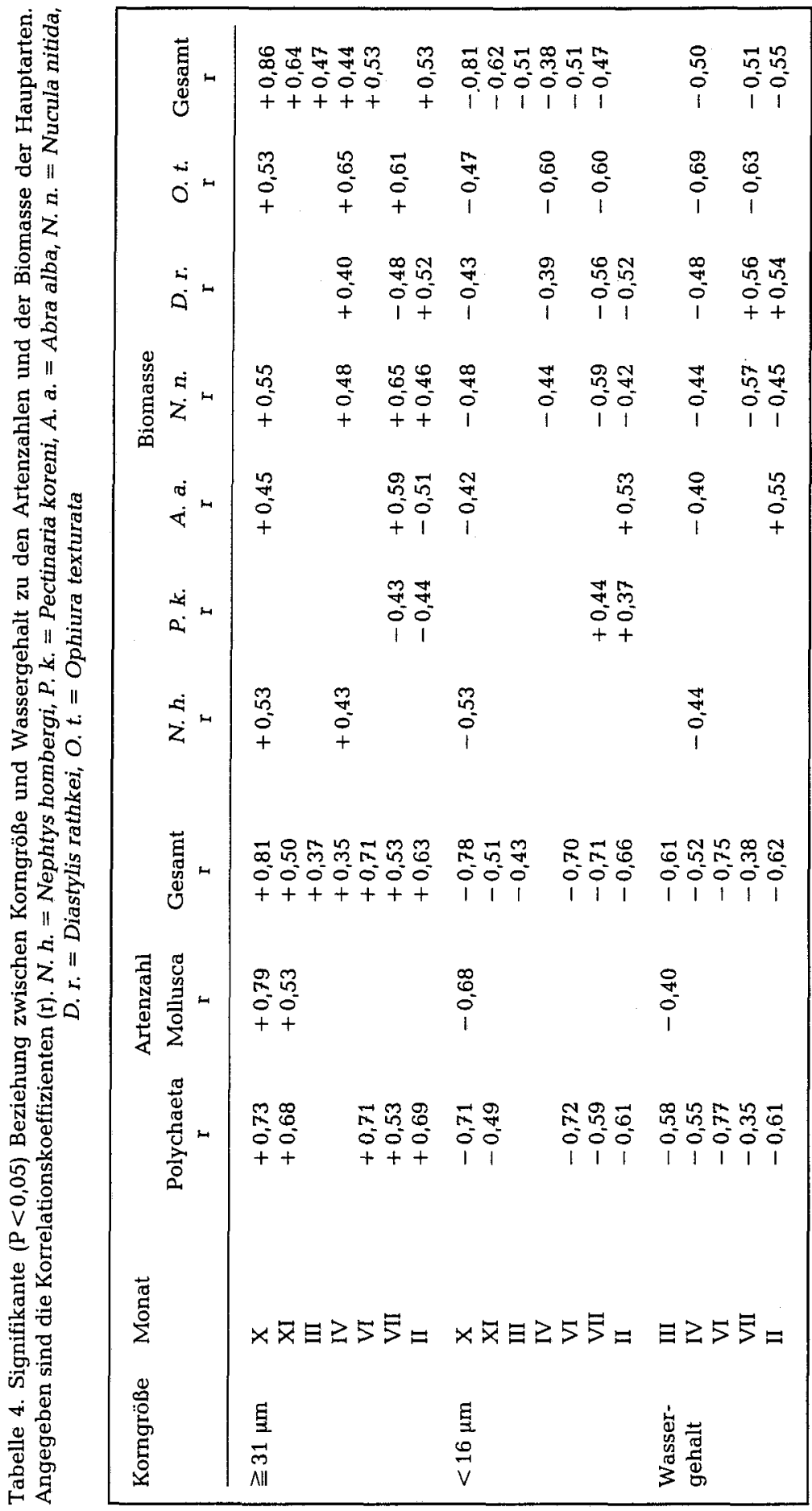


Wetterlagen begünstigt, wird der hohe Schluff- und Wassergehalt im Sediment bestimmter Zonen aufgebaut, die die siedelnden Arten nach Möglichkeit meiden.

Wie auch für die Artenzahl wurde für die Biomasse die Korrelation zu den Sedimenteigenschaften Korngröße und Wassergehalt geprüft (Tab. 3). Die Biomasse der häufigsten Arten war meist positiv mit den gröberen Korngrößen korreliert und negativ mit dem Wassergehalt. Wie an der Höhe des Korrelationskoeffizienten $r$ zu erkennen war, wirken auf die einzelnen Arten verschiedene Bereiche des Korngrößenspektrums. Nephtys hombergi und Pectinaria koreni hatten die höchste Korrelation mit den Korngrößenanteilen zwischen 8 und $4 \mu \mathrm{m}$, Nucula nitida und Ophiura texturata hingegen mit den Korngrößen von mehr als $31 \mu \mathrm{m}$. Die Gesamtbiomasse wurde am stärksten durch den Anteil der Korngröße $>31 \mu \mathrm{m}$ beeinflußt.

Für das abweichende Verhalten der Arten Abra alba und Diastylis rathkei gegenüber den Sedimentfraktionen im Vergleich der einzelnen Monate bietet sich folgende Erklärung: Da sich die Präferenz einer Art für eine bestimmte Korngröße im Jahresverlauf vermutlich nicht ändert, kann die Ursache für die geringe Biomasse von A. alba im Winter an Stationen mit hohem Anteil gröberen Sedimentes durch eine erhöhte Mortalität begründet werden. Für die hohe Sterblichkeit könnten Räuber (Fische, Echinodermata) verantwortlich sein. Diese meiden offensichtlich Gebiete mit hohem Anteil von Feinstsediment, in denen $A$. alba den Winter überlebte.

Diastylis rathkei zeigte mit Ausnahme von Juli 1980 eine positive Relation zu den grobkörnigen Korngrößen. Im Sommer (Juli 1980) hatte diese Art hingegen negative Beziehungen zu den mittleren Schluff-Fraktionen und positive zu den sehr feinen Anteilen. Dieses Phänomen ist im wesentlichen mit dem Massenauftreten von $A$. alba zu erklären. A. alba bevorzugt die gröberen Anteile im Sediment. Dort, wo A. alba in großen Mengen auftrat, mußte Diastylis rathkei aus Raummangel auf die feineren Sedimente ausweichen.

\section{Diversität}

Eine quantitative Beschreibung für den Artenreichtum eines Ökosystems ist die Diversität, die durch verschiedene Indices berechnet werden kann: Shannon-WienerIndex $\left(\mathrm{H}^{\prime}\right)$ und eveness $(\mathrm{J})$, bzw. das Verhältnis von Individuenzahl $(\mathrm{N})$ und Artenzahl (S), das die mittlere Individuenzahl pro Art (N/S) kennzeichnet. Die Empfindlichkeit verschiedener Indices gegenüber Schwankungen der Arten- und Individuenzahl wurde geprüft.

Erwartet wurde eine geringe Diversität, bzw. hohe Dominanz bei den belasteten Stationen 3 und 4 und eine hohe Diversität an den Stationen 1 und 2 und den Vergleichsstationen. Eine niedrige Diversität im Frühjahr auf Grund von Larvenfall, hingegen eine erhöhte Diversität im Herbst auf Grund niedriger Individuendichte wurde ebenfalls vermutet.

Der Shannon-Wiener-Index und eveness $\left(\mathrm{H}^{\prime}\right.$ und $\left.\mathrm{J}\right)$ sind stets zusammen anzugeben (Gray, 1981) und zeigten im vorliegenden Fall (Abb. 8) starke Schwankungen im Jahresgang und keinen einheitlichen Trend für die Dauerstationen. Bemerkenswert sind die Einbrüche in der Diversität bei Massenentwicklungen von Abra alba, Pectinaria koreni (Juni, Juli 1980) und Diastylis rathkei (April 1981). Die Vergleichsstationen hatten allgemein eine höhere Diversität als die Dauerstationen. Innerhalb der Dauerstationen lag die Diversität an der Station 1 über der der anderen Stationen, und die eveness war 

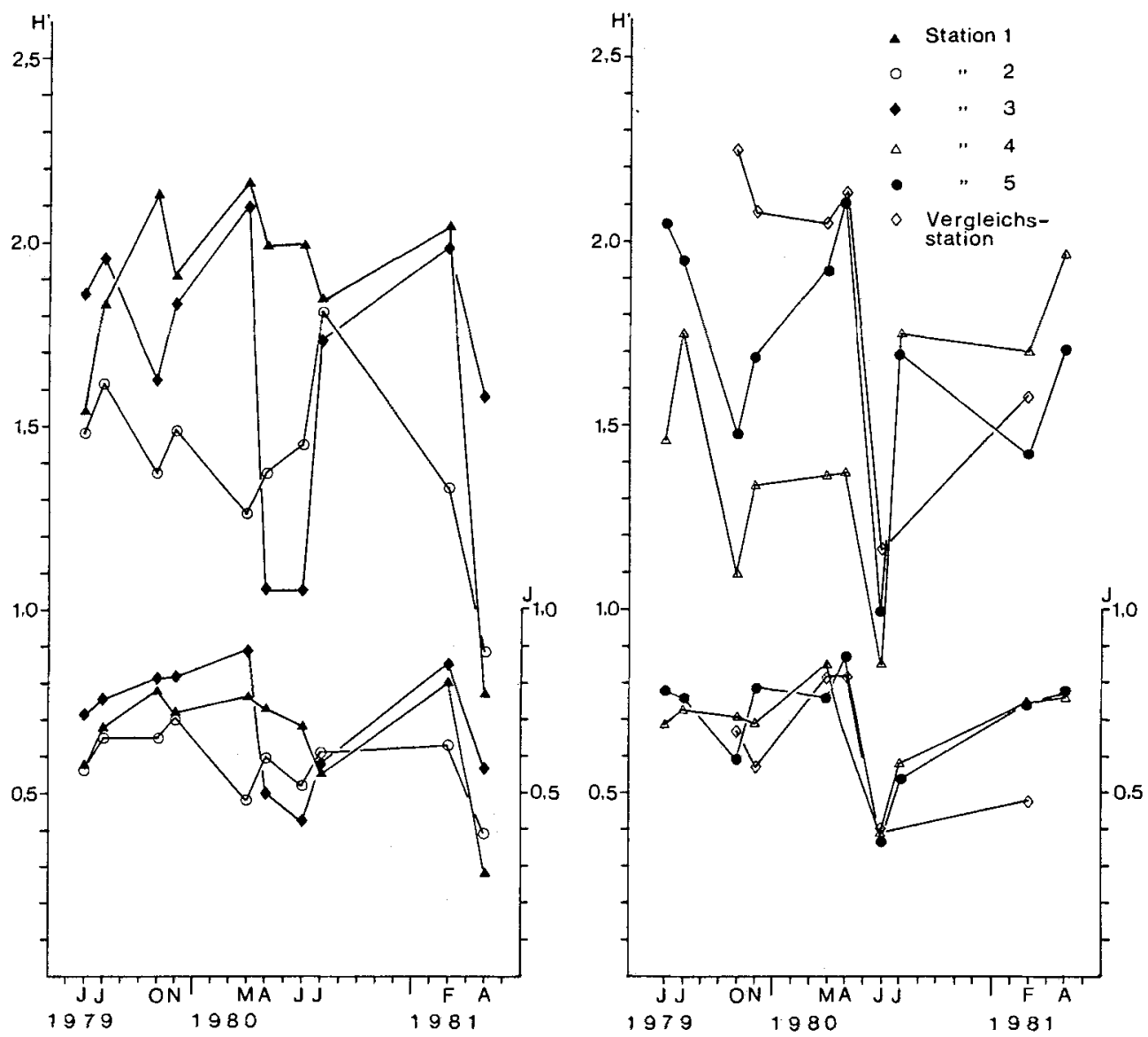

Abb. 8. Jahresgang der Diversitätsindices $\mathrm{H}^{\prime}$ (Shannon-Wiener-Index) und $J$ (eveness) an den Dauerstationen und den Vergleichsstationen

gleichmäßig hoch. Im Mittel über den Untersuchungszeitraum zeigte die Station 2 die geringste Diversität und eveness.

Mittlere Individuendichte pro Art (N/S): Ein niedriger N/S Index bedeutet in der Regel eine hohe Diversität. Im Jahresverlauf zeichnete sich der gleiche Trend an allen Dauerstationen ab, der vom Spätsommer bis zum Frühling (April) ein gleichbleibendes Verhältnis zeigte (Abb. 9). Erst in einer kurzen Periode vom Frühsommer bis Hochsommer wurde es durch hohe Werte unterbrochen.

Im allgemeinen war die N/S Relation hoch an Station $3(39,4)$ und Station $1(27,1)$, niedrig aber an Station $4(19,8)$. Ein bemerkenswertes Ergebnis, da ein niedriger Index eine hohe Artenzahl vermuten läßt. Es war jedoch gerade an Station 4 die minimale Artenzahl im Vergleich der Dauerstationen zu finden. Hier wurde trotz geringer Artenzahl auf Grund der ebenfalls minimalen Individuenzahl der geringe N/S Wert errechnet. An Station 1 ergab sich andererseits trotz hoher Artenzahl ein hoher N/S Index, bedingt durch eine hohe Individuenzahl. 

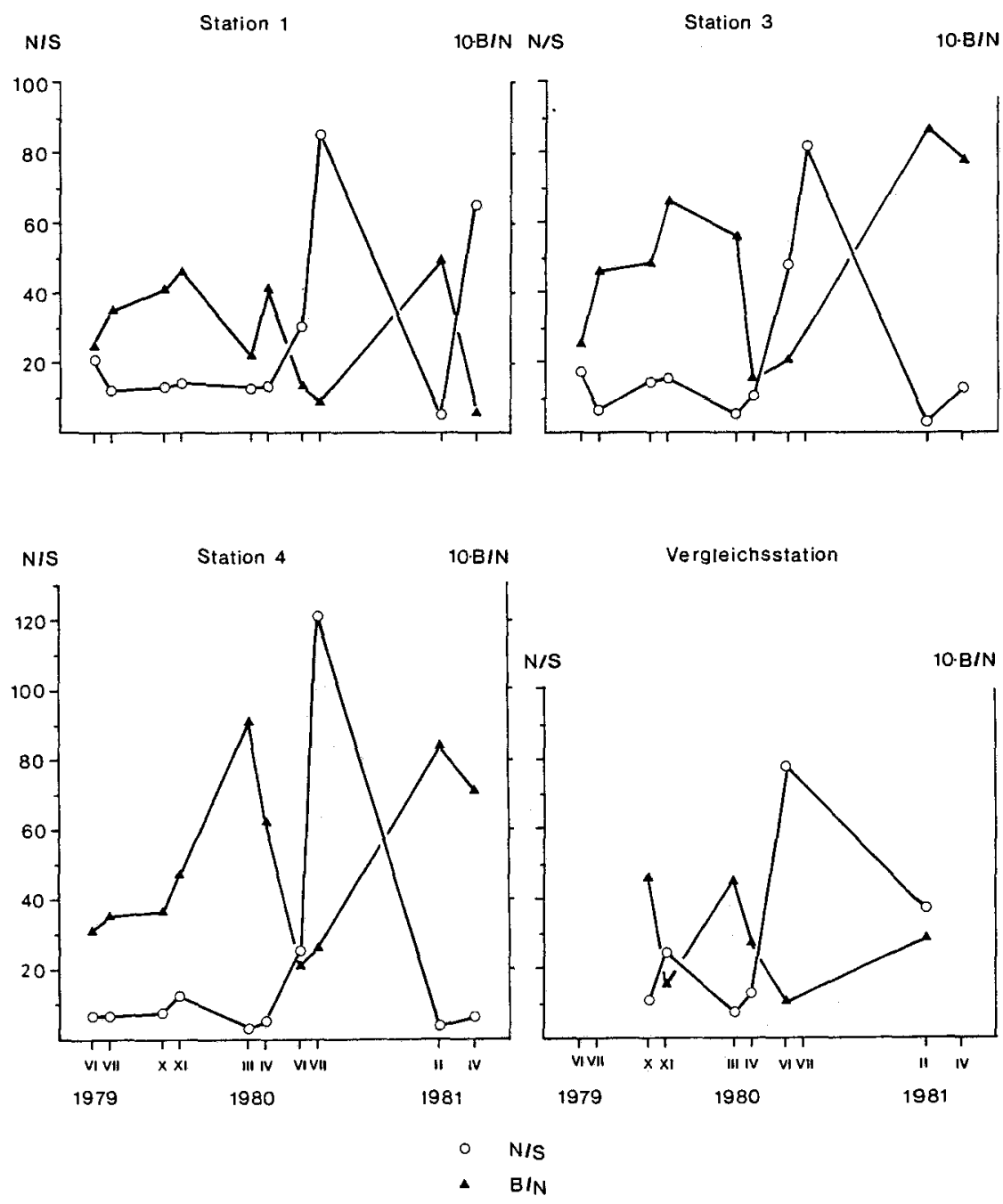

Abb. 9. Jahresgang der Indices N/S und B/N am Beispiel der Stationen 1, 3, 4 und den Vergleichsstationen (die $\mathrm{B} / \mathrm{N}$ Skala ist zehnfach überhöht dargestellt)

Häufig sind in ungestörten Gemeinschaften viele Arten durch ein oder weniger Individuen repräsentiert (Blackstock et al., 1986). Die Verteilung der Individuen auf die Arten ist dann log-normalverteilt (Gray \& Pearson, 1982; Pearson et al., 1983). Eine Störung durch organisch reiches Material bewirkt einen Anstieg der Individuenzahl bei wenigen, toleranten Arten, was ein Abknicken der log-Normalverteilungskurve zur Folge hat (Gray, 1979; Gray \& Mirza, 1979; Gray \& Pearson, 1982). Eine länger andauernde Störung würde ein Abflachen der Verteilungskurve zur Folge haben. Die Steigung der Verteilungskurve ist also ein Diversitätsmaß, wobei Steigung und Diversität einander proportional sind. 
Für den vorliegenden Fall wurde somit eine geringe Steigung der Verteilungskurve an den belasteten Stationen und eine hohe Steigung der Kurve bei den relativ unbelasteten Stationen 1 und 2 erwartet. Am Beispiel der Stationen 1 und 4 im Jahresgang zeigt sich (Abb. 10), daß nur an Station 1 die Werte graphisch an eine Gerade angepaßt werden können. Mittels einer Regressionsanalyse konnte auch für die übrigen Stationen eine Gerade erstellt werden, und die Steigung der berechneten Geraden, also die Diversität, jeder Dauerstation wurde für jede Jahreszeit berechnet. Es ergab sich durch eine Kovarianzanalyse kein signifikanter Unterschied $(P<0,05)$ zwischen den Steigungen der Verteilungskurven der Stationen 1 und 4.

Allen drei Methoden (Shannon-Wiener-Index, eveness, N/S) war gemeinsam, daß sie für den Sommer eine Dominanz einer oder weniger Arten dokumentieren. Während aber der Shannon-Wiener-Index und die eveness starken Schwankungen unterliegen, weist der N/S Index nur geringe Fluktuationen auf. Zur Zeit der Massenentwicklung weniger Arten zeigt aber der N/S Index einen extrem hohen Anstieg, während $\mathrm{H}^{\prime}$ und $J$ nicht so drastisch reagieren. Das ist durch die logarithmische Transformation der Werte bei der Berechnung von $\mathrm{H}^{\prime}$ und $\mathrm{J}$ begründet. Hierdurch werden die Extremwerte nivelliert.

Für eine schnelle Überprüfung der jahreszeitlichen Veränderung in Bodentiergemeinschaften ist der N/S Index geeignet, da er weniger aufwendig ist als die Berechnung von $\mathrm{H}^{\prime}$ und $\mathrm{J}$. Wenig hilfreich hat sich die graphische Methode über die log-Normalverteilung erwiesen. Sie soll nach Gray (1979) und Mirza \& Gray (1981) empfindlich für die Dokumentation von Störungen sein. Nach den eben genannten Ergebnissen wäre nach dieser Methode die Station 4 vergleichbar der Station 1, ohne den großen Unterschied in der Artenzahl beider Stationen zu berücksichtigen. Daraus ist zu folgern, daß die logNormalverteilung allein nicht als Indikator für die Ausgeglichenheit eines Systems ausreicht.

\section{Das mittlere Individualgewicht}

Das Verhältnis der Biomasse zur Individuenzahl gibt das mittlere Gewicht eines Tieres an. Der B/N Index der vorliegenden Untersuchung (Abb.9) war im frühen Sommer nach erfolgtem Larvenfall niedrig. Durch Absterben einiger Individuen einerseits und Wachstum andererseits stieg er zum Herbst an. Im Frühjahr fiel er durch hohe Individuendichten infolge von Larvenfall wieder ab.

Während an den Stationen 3 und 5 die Abnahme des B/N Index in erster Linie durch Biomasseverluste zu erklären ist, nahm an Station 4 die Relation trotz eines Biomassenanstiegs im Juni $1980 \mathrm{ab}$. Dies wurde bedingt durch eine entsprechend große Individuenzunahme. An Station 3 war der Biomassezuwachs größer als die Individuenzunahme, weshalb hier der Index stieg. Für den Winter (Februar 1981) wurde ein hoher $\mathrm{B} / \mathrm{N}$ Index berechnet, was durch ein Absterben vieler Individuen und dem Überleben weniger, großer Tiere begründet wird. Zwar war im April ein Anstieg der Biomasse zu messen, doch traten derart viele Jungtiere auf, daß die B/N Relation im Vergleich zu Februar fiel.

Die berechneten B/N Relationen unterlagen also einer deutlichen saisonalen Fluktuation. Eine große Fluktuation des Index ist vor allem bei Beständen mit einem hohen Anteil von einjährigen Arten zu erwarten. Hingegen ist bei einer Dominanz von mehrjährigen Arten eine geringe Schwankung des B/N Index zu erwarten, weil hier eine 

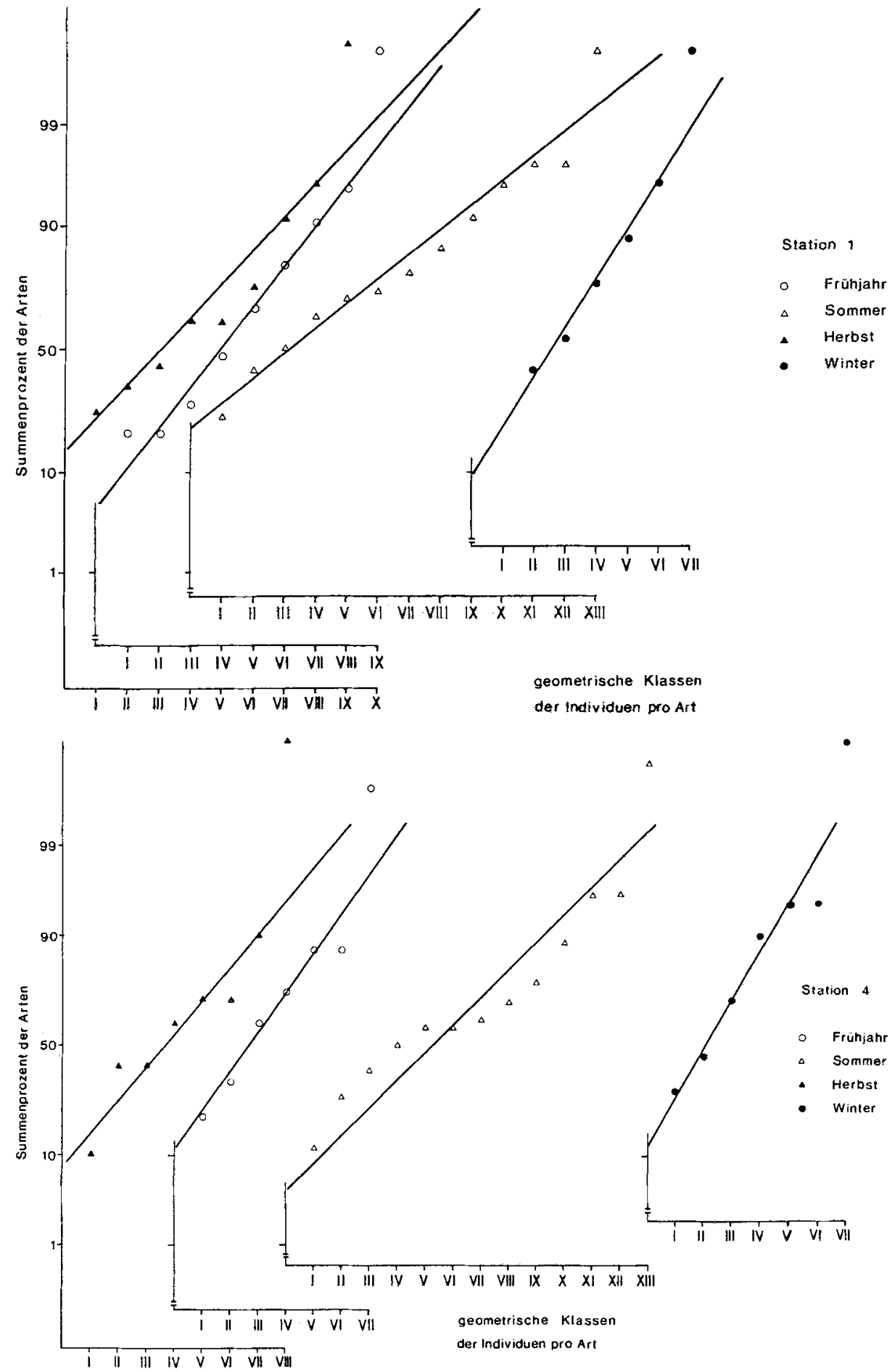
annähernd gleichbleibend hohe Biomasse zu messen sein wird. Hierbei wird die niedrige Biomasse vieler Jungtiere (kleiner B/N Index) durch die Anwesenheit weniger schwerer Tiere (hoher B/N Index) ausgeglichen.

Das erscheint besonders im vorliegenden Fall wichtig, da sich auf Grund des stark schwankenden $\mathrm{B} / \mathrm{N}$ Wertes auf eine Zusammensetzung von überwiegend ein- bis wenigjährigen Arten schließen läßt. Nur an Station 1 waren durch die Dominanz langlebiger Arten (Nucula nitida) die Schwankungen im B/N Index schwächer ausgeprägt.

Hohe Schwankungen der B/N Relation, wie sie an Station 4 auftraten, deuten also auf eine Population kurzlebiger Arten hin. Auch Pearson et al. (1982) geben hohe Schwankungen im B/N Index für Gebiete mit Belastung durch organische Substanz an, während die entsprechende Vergleichsstation - wie im vorliegenden Fall - nur geringe Schwankungen zeigt. Rapport et al. (1981) halten ebenfalls zunehmende Populationsschwankungen in Raum und Zeit für ein Streß-Symptom eines Ökosystems.

Die B/N Relation ist also ein schnell durchzuführendes, wenn auch grobes Maß für die Stabilität eines Systems. Hiernach ist an den Stationen 3,4 und 5 auf ein weniger konstantes System zu schließen.

\section{DISKUSSION}

Der beobachtete jahreszeitliche Trend in Arten- und Individuenzahl mit minimalen Werten im Winter kann durch Wintermigration verursacht sein, wie Beukema et al. (1978) für Arten des Eulitorals beschreiben. Eine weitere Ursache für den jahreszeitlichen Wechsel in Arten- und Individuenzahl kann das Absterben einjähriger Arten im Winter sein. Beeinflussen doch extrem niedrige Temperatúren, wie sie im Winter 1978/79 vorlagen, die Fauna des Sublitorals, wobei vor allem die Mollusca und Echinodermata empfindlich reagieren. Ausnahmen sollen die Arten Nucula nitida und Ophiura texturata sein (Ziegelmeier, 1964; Dörjes, 1980). Als relativ unempfindlich bezeichnet Ziegelmeier (1964) Crustacea und Polychaeta. Unter ihnen scheinen die kleinen Formen am widerstandsfähigsten zu sein. Crisp (1964) führt das Absterben vieler Arten bei sehr kalten Temperaturen auf das Herabsetzen der Körperaktivitäten wie Befreien der Cilien von Feinstpartikeln, Festhalten am Untergrund, Graben etc. zurück.

Neben der geringen Artenzahl im Winter fiel an den Dauerstationen die Station 4 durch eine permanent geringe Artenzahl auf. Die minimale Artenabundanz an dieser Station wurde schon 1978 registriert und als deren Ursache ein Einfluß von Klärschlamm angenommen (Mühlenhardt-Siegel, 1981). Die negative Wirkung des Klärschlamms kann in der Bildung von $\mathrm{H}_{2} \mathrm{~S}$, der regelmäßigen Überdeckung oder dem Eintrag von sehr feinkörnigem, wasserreichen Sediment zu finden sein. Auch Pearson \& Rosenberg (1976, 1978) beobachteten geringe Artenzahlen in Gebieten unter ökologischem Streß im Sinne von Gray (1981, 1982). Anders als in den meisten gestreßten Gebieten nahm die Individuendichte mit der Artenzahl ab, eine Erscheinung, die nach Pearson \& Rosenberg (1978) erst in "grossly polluted" Gebieten auftritt.

Abb. 10. Verteilung der Individuen auf die Arten nach der graphischen Methode für die Dauerstationen 1 und 4 
Die Reduzierung der Artenzahl in belasteten Gebieten ist ein weit verbreitetes Phänomen und wurde von Tulkki (1968) für die finnische Küste im Bereich KotkaHelsinki-Turku-Pori beschrieben, für die Deutsche Bucht von Caspers (1979), McIntyre (1978) und Rachor (1977, 1980, 1982). Minimale Artenzahlen in verunreinigten Meeresgebieten beobachteten ebenfalls Pearson $(1971,1975,1980,1981,1982)$ und Topping \& McIntyre (1972) in schottischen und Parker (1980) in irischen Fjorden. Auch in gestreßten Seegebieten vor Cornwall (Probert, 1981) und Portsmouth (Soulsby et al., 1978) lagen geringe Artenzahlen vor, weiterhin im Oslofjord (Gray \& Mirza, 1979; Mirza \& Gray, 1981) und dem Saltkällefjord und Seegebieten West-Schwedens (Tulkki, 1968; Rosenberg, 1971, 1974, 1977, 1980). Eine Reduzierung der Artenzahl wurde von Stirn (1973) für belastete Teile des Mittelmeeres, wie auch für die New Yorker Bucht von Young \& Pearce (1975) und Botton (1979) beschrieben. Gleiches berichtet Reish (1980) für das Seegebiet vor Kalifornien. Eleftheriou et al. (1982) analysierten im Experiment eine Verminderung der Artenzahl bei Verunreinigung mit Klärschlamm. Allein Dauer \& Connor (1980) untersuchten neben dem Anstieg von Biomasse und Individuenzahl auch die Zunahme der mittleren Artenzahl unter dem Einfluß von Klärschlamm auf Sandsedimenten eines Seegebietes vor Florida.

Die Individuendichte zeigte eine saisonabhängige Tendenz, wie sie auch von anderen Seegebieten berichtet wurde: Ostsee (Kühlmorgen-Hille, 1963; Persson, 1978), Nordsee (Ziegelmeier, 1978), Atlantikküste der USA (Steimle, 1982), Delaware Bay, USA (Maurer et al., 1978). Die Jahresmittelwerte lagen im Untersuchungsgebiet zwischen 551 Ind. $/ \mathrm{m}^{2}$ und $2219 \mathrm{Ind} . / \mathrm{m}^{2}$. Die sehr hohe Dichte von $3828 \mathrm{Ind} . / \mathrm{m}^{2}$, die Salzwedel et al. (1985) als Mittelwert für die "Nucula nitidosa-Assoziation" im gleichen Untersuchungsgebiet für den Herbst angeben, wurde im Herbst 1979 mit $2240 \mathrm{Ind} . / \mathrm{m}^{2}$ in der vorliegenden Untersuchung nicht erreicht. Ein Vergleich mit anderen Seegebieten oder älteren Untersuchungen in der Nordsee ist wegen der früher benutzten gröberen $(1 \mathrm{~mm})$ Maschenweite der Siebe nicht möglich.

Es zeigte sich eine erhöhte Individuenzahl auf Sandsedimenten gegenüber Schlickgrund. Für Sandsedimente bei Cape Cod in 15 bis $20 \mathrm{~m}$ Tiefe geben Young \& Rhoads (1971) eine maximale Dichte von 30150 Ind. $/ \mathrm{m}^{2}$ und eine mittlere Dichte von 15410 Ind./ $\mathrm{m}^{2}$ an (Maschenweite $1 \mathrm{~mm}$ ). Auch Vanosmael et al. (1982) nennen für Sandsedimente (8-10 m Tiefe) mittlere Werte von $4910 \mathrm{Ind} . / \mathrm{m}^{2}$, arbeiteten allerdings mit $0,25 \mathrm{~mm}$ Maschenweite. Für "muddy sand" geben Maurer et al. (1978) 222,4 bis 1217,5 Ind./m² an (Maschenweite $1 \mathrm{~mm}$ ). Andererseits weist Dörjes (1977) eine deutliche Individuenabnahme von feinen zu groben Sedimenten nach. Maurer et al. (1978) schränken dies ein, indem sie einen Anstieg der Individuenzahl bei mittleren Korngrößen analysierten. Die vorliegende Untersuchung zeigte ebenfalls ein Meiden der feinkörnigen Substrate in Nähe der Klärschlammeinbringung seitens der Bodentiere.

Die Biomasse im Untersuchungsgebiet unterlag jahreszeitlichen Schwankungen, wie sie auch für andere Küstengebiete beschrieben wurden: Kieler Bucht (Arntz, 1971a; Brunswig et al., 1976), Northumberland, England (Buchanan \& Warwick, 1974), Deutsche Bucht (Stripp, 1969), Eulitoral der Niederlande (Beukema, 1974). Die Jahresmittelwerte der Biomasse an den Dauerstationen erreichten maximal 7,02 g AFTG/m ${ }^{2}$ (Station 1) und minimal $3,12 \mathrm{~g} \mathrm{AFTG} / \mathrm{m}^{2}$ (Station 4). Hierbei blieben die Werte der Massenentwicklung unberücksichtigt.

Die gefundenen Werte lagen im Bereich der Ergebnisse, die Buchanan \& Warwick 
(1974) in Northumberland (3,4-3,8 $\mathrm{g}$ AFTG/m ${ }^{2}$ im Januar bis April und 4,2-4,5 g AFTG/ $\mathrm{m}^{2}$ von Juni bis November) erhielten und denen von Maurer \& Aprill (1979) im Seegebiet von Delaware, USA $\left(0,47-9,6 \mathrm{~g} \mathrm{AFTG} / \mathrm{m}^{2}\right)$. Nur geringfügig höhere Werte geben Arntz \& Brunswig (1975) für die Kieler Bucht an (7,9 g AFTG/m²) sowie Bloom et al. (1972) für die Ostküste der USA $\left(7,68 \mathrm{~g} \mathrm{AFTG} / \mathrm{m}^{2}\right)$. Weit höhere Werte wurden als Vergleich dazu in der Macoma-Gesellschaft im Wattenmeer der Niederlande mit 18,4-20,5 g AFTG/m² gemessen (Beukema et al., 1978). Geringere Biomasse als im Untersuchungsgebiet fanden Lie \& Kisker (1970) im Puget Sound, USA mit 1,398-3,058 g AFTG/m².

Die Höhe der Biomasse läßt also keine Schlüsse auf den Grad einer Belastung durch Streß zu. Hingegen war das Verhältnis B/N gut geeignet als ein - wenn auch grobes $\mathrm{Maß}$ für die Stabilität eines Systems. Wie auch an den hier untersuchten Dauerstationen unterlag im belasteten System von Loch Eil der B/N Index hohen Schwankungen (Pearson et al., 1982). Hohe saisonale Schwankungen im B/N Index werden durch die Dominanz kurzlebiger Arten verursacht, wie an den Dauerstationen in der Nähe des Verklappungsgebietes zu erkennen war. Als Beispiel sind hier die Arten Diastylis rathkei, Pectinaria koreni und Scalibregma inflatum (Hagmeier, 1925) zu nennen. Auch Abra alba muß in diesem Gebiet als kurzlebig bezeichnet werden.

Von den Diversitätsindices reagierte der Index N/S empfindlich auf starke Veränderungen in der Bestandsdichte. Über hohe Schwankungen des Index N/S an Stationen des organisch belasteten Fjords Loch Eil in Schottland berichten Pearson et al. (1982). Im Gegensatz hierzu bestanden geringe Schwankungen an der entsprechenden Vergleichsstation in Loch Linnhe. Rapport et al. (1981) halten zunehmende Populationsschwankungen für ein Streß-Symptom eines Ökosystems, das durch Auftreten kurzlebiger Arten (rStrategen), die typische Besiedler belasteter Gebiete sind, verursacht wird (Rapport et al., 1981; Pearson, 1980; Gray, 1982). Nach McCall (1977)' treten r-Strategen häufig bei instabilen Sedimenten mit hohem Wassergehalt auf, wie sie hier an den meisten Dauerstationen vorlagen.

Die Berechnung der Diversitätsindices und deren Veränderung im Jahresgang erwies sich also als hilfreich, um Störungen zu erkennen. Nur Godfrey (1978) hält Diversitätsindices als Anzeiger von Verschmutzungseffekten für ungeeignet, weil die taxonomische Zusammensetzung der Gesellschaft unberücksichtigt bleibt, die sicherlich von Bedeutung ist.

Nach Gray $(1979,1981)$ und Gray \& Mirza (1979) ist die Prüfung auf log-Normalverteilung der Individuen auf die Arten eine gute Methode, den Einfluß von Streß zu erkennen. Für die vorliegende Untersuchung konnte sie jedoch nicht erfolgreich angewendet werden. Die Schwierigkeiten ergaben sich durch die Ungenauigkeit der zeichnerischen Methode, bzw. bei der Transponierung der Werte aus dem Wahrscheinlichkeitsnetz in das lineare System. Wegen der genannten Ungenauigkeiten mußten gerade die Werte der hohen geometrischen Klassen wegfallen, die aber nach Gray $(1979,1981)$ maßgebend für die Indikatoreigenschaft dieser Methode sein sollen. Unterschiede in der Steigung der Summenprozentkurven, wie sie nach Gray \& Mirza (1979) zwischen gestreßten und unbelasteten Gebieten auftreten sollen, ließen sich hier an den Dauerstationen nicht nachweisen. Diese Tatsache kann mit der Darstellung als Summenprozentkurve begründet werden. Die Methode hat meines Erachtens als Indikator für Verschmutzung ihre Grenze in dem Bereich, der nach Pearson \& Rosenberg $(1976,1978)$ zwischen "polluted" und "grossly polluted" bezeichnet wird, nämlich dort, wo geringe 
Artenzahlen bei geringer Individuendichte vorliegen. Shaw et al. (1983) und Lambshead \& Platt (1985) weisen auf die Unzulänglichkeit der graphischen Methode nach Gray $(1979,1981)$ hin. Sie bezeichnen das Abknicken der Summenprozentkurve bei Proben aus belasteten Gebieten als statistisch nicht gesichert und verwerfen diese Methode als Indikator für Streß. Shaw et al. (1983) ziehen die Dominanzverteilungskurve zur Darstellung der Verteilung von Individuen auf die Arten der log-Normalverteilung nach Gray $(1979,1981)$ vor, was nach eigenen Untersuchungen unterstützt werden muß (Abb. 11).

Station 1
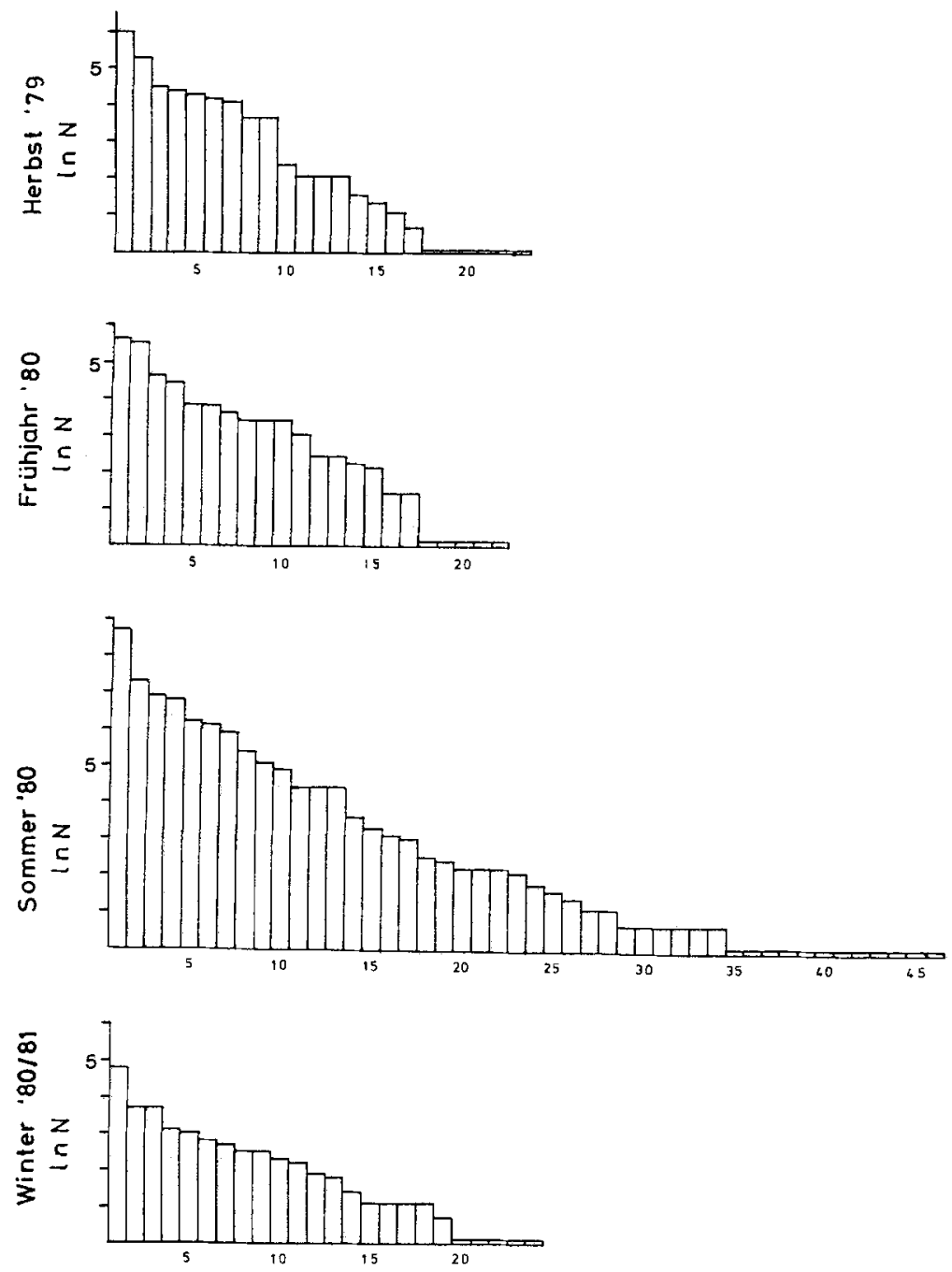

Abb. 11a. Dominanz-Diversitätsverhältnisse (Arten gerankt nach Individuenzahl N) am Beispiel der Dauerstation 1 für die unterschiedlichen Jahreszeiten Herbst (November 1979), Frühjahr (April 1980), Sommer (Juli 1980) und Winter (Februar 1981) 


\section{Station 4}
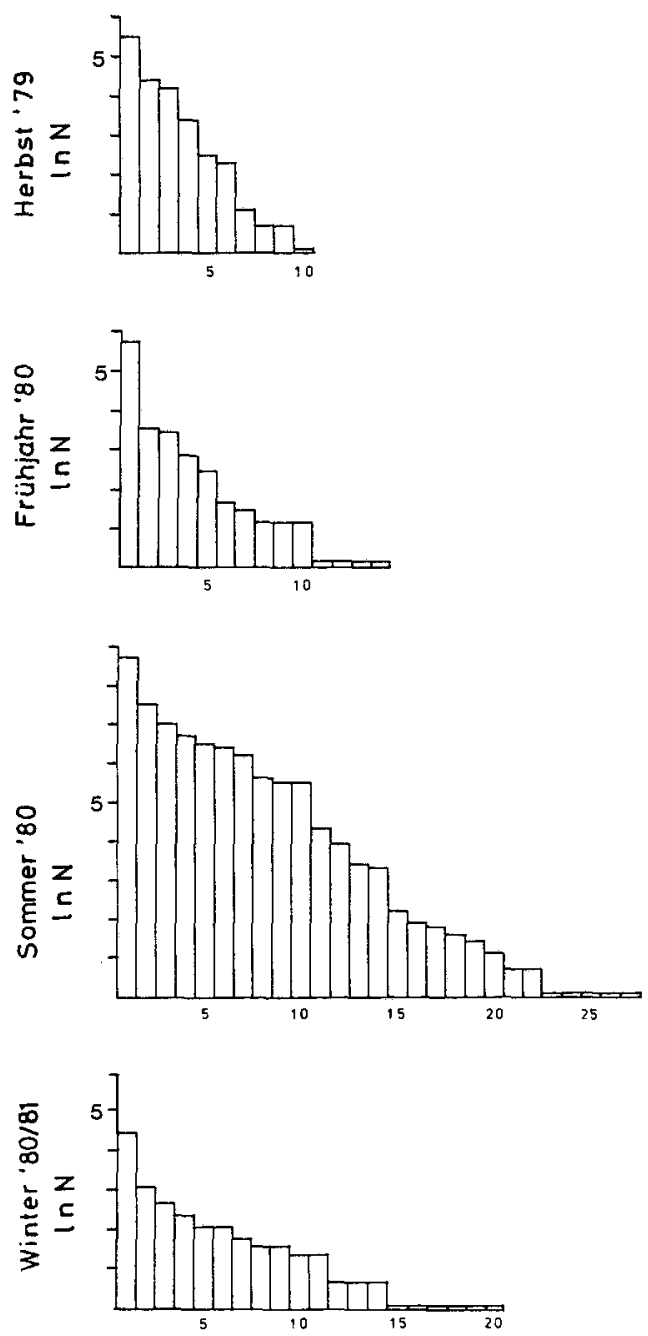

Abb. 11b. Dominanz-Diversitätsverhältnisse am Beispiel der Station 4

Besonders auffallend war im Juli 1980 die Massenentwicklung von Abra alba, Ensis directus und einiger Polychaeta, unter anderem Pectinaria koreni. Die hohe Individuendichte von Ensis directus wurde an anderer Stelle (Cosel et al., 1982; Mühlenhardt-Siegel et al., 1983) diskutiert. Die Ursache von Massenentwicklungen ist nach Ziegelmeier (1970) im Fehlen von Feinden zu suchen. Die Massenentfaltung von $A$. alba und die hohen Dichten von P. koreni waren auf Stationen in der Nähe des Klärschlammverklappungsgebietes beschränkt. Auch Caspers $(1978,1979)$ berichtet von einer großen Dichte der beiden Arten in den Jahren 1973/74 im gleichen Gebiet. Ein kausaler Zusammen- 
hang zwischen der Einbringung von Klärschlamm und der temporären Massenentwicklung weniger Arten erscheint fraglich, da schon zur Zeit vor der Verklappung über kurzzeitige Massenentwicklungen von $A$. alba berichtet wurde (Eisma, 1966). Neben einem adäquaten, weil schlickigem Substrat (Eisma, 1966; Bachelet, 1981) ist vermutlich eine günstige Nahrungsbasis für diese Arten als Detritusfresser durch die Verklappung und die Lage im Elbe-Ästuar gegeben. Da das beschriebene Areal nur dünn besiedelt war, konnten im Frühjahr 1980 nach dem Larvenfall sehr viele Jungtiere überleben, ohne daß die Räuber - z. B. Ophiura texturata und O. albida (Tyler, 1977) - sie dezimierten. Das erfolgreiche Siedeln von $A$. alba in einem Gebiet, das minimale Arten- und Individuenzahlen aufwies, bestätigt die Pioniereigenschaften dieser Art, die Glemarec \& Menesguen (1979) ihr zuschreiben. Andererseits hält Eagle (1975) die Freßaktivitäten von Abra und Pectinaria für eine Behinderung anderer Arten am Festsetzen. Mit ähnlicher Argumentation erklärt Caspers (1980) die minimalen Artenzahlen während der Massenentwicklung 1973/74.

Die vorliegende Analyse hingegen zeigte aber gerade eine erhöhte Artenzahl während der Massenentwicklung von $A$. alba an den Stationen im Einflußbereich der Klärschlammverklappung. Hierfür sind möglicherweise Sedimentveränderungen verantwortlich, die nach Rhoads (1974) und Jumars (1975) durch biologische Aktivitäten hervorgerufen werden. Soll doch gerade die Gattung Abra einen großen Beitrag zur Sedimentumlagerung leisten (Wikander, 1981). Im April 1981 war die Abra-Massenentwicklung weitgehend zusammengebrochen, die Art $P$. koreni fehlte fast vollständig im Untersuchungsgebiet. Ein Grund für den Zusammenbruch der Massenentwicklung mag in einer kurzen Lebensspanne der Art A. alba zu suchen sein.

A. alba wird in diesem Gebiet der Nordsee nur ein Jahr alt (10 bis 12 Monate), während ihr Alter von Glemarec \& Menesguen (1979) auf 14 Monate geschätzt wird. Hagmeier \& Künne (1951) und Arntz et al. (1976) bezeichnen 2 Jahre und HöpnerPetersen (1977) 4 Jahre als maximales Alter. P. koreni wird als einjährig angesehen, während andere Arten dieser Gattung mehrjährig sind (Nichols, 1974). Weitere Gründe für den Zusammenbruch von Massenentwicklungen sind Faktoren der Selbstregulation, z. B. Nahrungsmangel. Da seit Sommer 1980 kein Klärschlamm in diesem Gebiet mehr verklappt wurde, wäre die Nahrung als limitierender Faktor nicht auszuschließen. Da aber eine entsprechend starke Massenentwicklung von $A$. alba den Winter nicht überlebte (Caspers, 1978, 1979), obwohl die Nahrungszufuhr durch regelmäßige Verklappung erfolgte, bleibt diese Begründung für den Zusammenbruch der Massenentwicklung fraglich.

Darüber hinaus werden Massenentwicklungen schnell durch Predatoren kontrolliert. In diesem Falle sei auf die Zunahme von Ophiura texturata im April 1981 gegenüber Juli $1980 \mathrm{im}$ betreffenden Gebiet hingewiesen. Weitere Räuber von $A$. alba und $P$. koreni sind Bodenfische wie Scholle und Kliesche (Hertling, 1928; Johansen, 1928; Arntz, 1971b; Jones, 1952). Arntz \& Hempel (1972) geben $A$. alba und $P$. koreni unter anderen als wichtige Fischnahrung für Dorsche und Klieschen in der Deutschen Bucht an. Mit dem Zusammenbruch der Populationen von Abra und Pectinaria war ebenfalls die Zahl der übrigen Arten in dem genannten Gebiet wieder gefallen.

Die erniedrigten Artenzahlen, die temporären Massenentwicklungen von Pionierarten, die hohen saisonalen Schwankungen von Diversitätsindices wie Individuen pro Art $(\mathrm{N} / \mathrm{S})$, Shannon-Wiener-Index und eveness $\left(\mathrm{H}^{\prime}\right.$ und $\left.\mathrm{J}\right)$ und $\mathrm{B} / \mathrm{N}$ Index, bedingt durch die 
Dominanz kurzlebiger Arten an den Stationen im Einflußbereich der Klärschlammverklappung zeigen das Bild einer Bodentiergemeinschaft unter ökologischem StreB. Hierbei scheint der hohe Anteil der feinsten Korngrößen ein wichtiger Streßfaktor zu sein, der durch die Einbringung feinster Partikel mit dem Klärschlamm verstärkt wird.

Danksagungen: Mein Dank gilt in erster Linie Herrn Prof. Dr. C. D. Zander für seine Diskussionsbereitschaft und kritische Durchsicht des Manuskripts. Herrn Dr. E. Rachor, seinen Mitarbeitern und der Besatzung des FS "Victor Hensen" (Bremerhaven) und Herrn Dr. A. Albrecht, seinen Mitarbeitern und der Besatzung des FS "Gauss" (Hamburg) danke ich für die Möglichkeit der Teilnahme an den Seereisen und der großen Hilfe an Bord. Für die Überlassung von Sedimentdaten und der Möglichkeit, Sedimentanalysen durchzuführen, möchte ich mich bei der Abteilung M 33 des Deutschen Hydrographischen Instituts (Hamburg) bedanken. Frau Dr. B. Hilbig half mir bei den Fahrten im Juli 1980 und April 1981. Frau U. Harm tippte das Manuskript. Herr Dr. D. Bürkel korrigierte den englischen Text. Thnen sei ebenfalls herzlich gedankt.

\section{LITERATUR}

Arntz, W. E., 1971a. Biomasse und Produktion des Makrobenthos in den tieferen Teilen der Kieler Bucht im Jahr 1968. - Kieler Meeresforsch. 27, 36-72.

Arntz, W. E., 1971b. Die Nahrung der Kliesche (Limanda limanda, L.) in der Deutschen Bucht. - Ber. dt. wiss. Kommn Meeresforsch. 22, 129-183.

Arntz, W. E. \& Brunswig, D., 1975. An approach to estimating the production of macrobenthos and demersal físh in a western Baltic Abra alba community. - Merentutkimuslait. Julk. 239, 195-205.

Arntz, W. E. \& Hempel, G., 1972. Biomasse und Produktion des Makrobenthos in der Kieler Bucht und seine Verwertung durch Nutzfische. - Verh. dt. zool. Ges. 65, 32-37.

Arntz, W. E., Brunswig, D. \& Sarntheim, M., 1976. Zonierung von Mollusken und Schill im Rinnensystem der Kieler Bucht (westliche Ostsee). - Senckenberg. marit. 8, 189-269.

Bachelet, G., 1981. Données préliminaires sur l'organisation trophique d'un peuplement benthique marine. - Vie Milieu 31, 205-213.

Beukema, J. J., 1974. Seasonal changes in the biomass of the macrobenthos of a tidal flat area in the Dutch Wadden Sea. - Neth. J. Sea Res. 8, 94-107.

Beukema, J. J., De Bruin, W. \& Jansen, J. J. M., 1978. Biomass and species richness of the macrobenthic animals living on the tidal flats of the Dutch Wadden Sea: long term changes during a period with mild winters. - Neth. J. Sea Res. 12, 58-77.

Blackstock, J., Johannessen, P. J. \& Pearson, T. H., 1986. Use of a sensitive indicator species in the assessment of biological effects of sewage disposal in fjords near Bergen, Norway.-- Mar. Biol. $93,315-322$.

Bloom, S. A., Simon, J, L. \& Hunter, V. D., 1972. Animal - sediment relations and community analysis of a Florida estuary. - Mar. Biol. 13, 43-56.

Botton, M. L., 1979. Effects of sewage sludge on the benthic invertebrate community of the inshore New York Bight. - Estuar. coast. mar. Sci. 8, 169-180.

Brunswig, D., Arntz, W. E. \& Rumohr, H., 1976. A tentative field experiment on population dynamics of macrobenthos in the Western Baltic. - Kieler Meeresforsch. (Sonderh.) 3, 49-59.

Buchanan, J. B. \& Warwick, R. M., 1974. An estimate of benthic macrofaunal production in the off shore mud of the Northumberland coast. - J. mar. biol. Ass. U. K. 54, 197-222.

Caspers, H., 1978. Ecological effects of sewage sludge on benthic fauna off the German North Sea coast. In: 8th International Conference on Water Pollution Research, Sydney 1976. Ed. by S. H. Jenkins. Pergamon Press, Oxford, 951-956.

Caspers, H., 1979. Die Entwicklung der Bodenfauna im Klärschlammverklappungsgebiet vor der Elbe-Mündung. - Arb. dt. FischVerb. 27.

Caspers, H., 1980. Long-term changes in benthic fauna resulting from sewage sludge dumping into the North Sea. - Wat. Sci. Technol. 13, 461-479.

Cosel, R. von, Dörjes, J. \& Mühlenhardt-Siegel, U., 1982. Die amerikanische Schwertmuschel Ensis 
directus (Conrad) in der Deutschen Bucht. I. Zoogeographie und Taxonomie im Vergleich mit den einheimischen Schwertmuschelarten. - Senckenberg, marit. 14, 147-173.

Creutzberg, F. \& Postma, H., 1979. An experimental approach to the distribution of mud in the Southern North Sea. - Neth. J. Sea Res. 13, 99-116.

Crisp, D. J., 1964. The effects of the winter $1962 / 63$ on the British marine fauna. - Helgoländer wiss. Meeresunters. 10, 313-327.

Dauer, D. M. \& Conner, W. G., 1980. Effects of moderate sewage input on benthic polychaete populations. - Estuar. coast. mar. Sci. 10, 335-346.

Deutsches Hydrographisches Institut, 1979. Beobachtungen auf den deutschen Feuerschiffen der Nord- und Ostsee sowie Monatsmittelwerte von Temperatur und Salzgehalt. - Meeresk. Beob. Ergebn. dt. hydrogr. Inst. 48, 1-157.

Deutsches Hydrographisches Institut, 1980. Beobachtungen auf den deutschen Feuerschiffen der Nord- und Ostsee sowie Monatsmittelwerte von Temperatur und Salzgehalt. - Meeresk. Beob. Ergebn. dt. hydrogr. Inst. 50, 1-134.

Deutsches Hydrographisches Institut, 1981. Beobachtungen auf den deutschen Feuerschiffen der Nord- und Ostsee sowie Monatsmittelwerte von Temperatur und Salzgehalt. - Meeresk. Beob. Ergebn. dt. hydrogr. Inst. 52, 1-134.

Deutsches Hydrographisches Institut, 1982. Beobachtungen auf den deutschen Feuerschiffen der Nord- und Ostsee sowie Monatsmittelwerte von Temperatur und Salzgehalt. - Meeresk. Beob. Ergebn. dt. hydrogr. Inst. 53, 1-128.

Deutsches Hydrographisches Institut, 1983. Beobachtungen auf den deutschen Feuerschiffen der Nord- und Ostsee sowie Monatsmittelwerte von Temperatur und Salzgehalt. - Meeresk. Beob. Ergebn. dt. hydrogr. Institut 54, 1-110.

Dörjes, J., 1977. Faunistic studies on Borkum Riffgrund (North Sea). - Senkenberg. marit. 9, 1-17.

Dörjes, J., 1980. Auswirkung des kalten Winters 1978/79 auf das marine Makrobenthos. - Senkenberg. marit. 374, 109-115.

Dybern, B. J., 1980. Pollution in the Baltic. In: Marine pollution and sea life. Ed. by M. Ruivo. Fishing News, London, 15-23.

Eagle, R. R. A., 1975. Natural fluctuations in a soft bottom benthic community. - J. mar. biol. Ass. U. K. 55, 865-878.

Eisma, D., 1966. The distribution on benthic marine molluscs of the main Dutch coast. - Neth. J. Sea Res. 3, 107-163.

Eleftheriou, A., Moore, D. C., Bosford, D. J. \& Robertson, M. R., 1982. Underwater experiments on the effects of sewage sludge on a marine ecosystem. - Neth. J. Sea Res. 16, 465-473.

Figge, K., 1981. Karte der Sedimentverteilung in der Deutschen Bucht mit Begleitheft. Deutsches Hydrographisches Institut, Hamburg.

Franz, E. H., 1981. A general formulation of stress phenomena in ecological systems. In: Stress effects on natural ecosystems. Ed by G. W. Barrett \& R. Rosenberg. Wiley, New York, 49-54.

Gadow, S. \& Schäfer, A., 1973. Die Sedimente der Deutschen Bucht: Korngrößen, Tonmineralien und Schwermetalle. - Senckenberg marit. 5, 165-178.

Glemarec, M. \& Menesguen, A., 1979. Functioning of a muddy sand ecosystem: seasonal fluctuations of different trophic levels and difficulties in estimating production of the leader macrofauna species. In: Production benthique dans le Golfe de Gascogne. Ed. par M. Glemarec. Inst. d'Etudes Marines, Brest, $11 \mathrm{pp}$.

Godfrey, P. J., 1978. Diversity is a measure of benthic macroinvertebrate community response to water pollution. - Hydrobiologia 57, 111-122.

Goedecke, E., 1954. Über Ergebnisse neuerer Untersuchungen der Temperaturverhältnisse in der Deutschen Bucht. - Ber. dt. wiss. Kommn Meeresforsch. 13, 283-297.

Gray, J. S., 1974. Animal - sediment relationships. - Oceanogr. mar. Biol, 12, 223-261.

Gray, J. S., 1979. Pollution induced changes in populations. - Philos. Trans. R. Soc. Lond. (B) 286, $545-561$.

Gray, J. S., 1981. The ecology of marine sediments. Ed. by R. S. K. Barnes, P. L. Miller, J. Paul \& T. ap Rees, Cambridge Univ. Press, Cambridge, 185 pp.

Gray, J. S., 1982. Effects of pollutants on marine ecosystems. - Neth. J. Sea Res. 16, 424-443.

Gray, J. S. \& Mirza, F. B., 1979. A possible method for the detection of pollution-induced disturbances on marine benthic communities. - Mar. Pollut. Bull. 10, 142-146. 
Gray, J. S. \& Pearson, T. H., 1982. Objective selection of sensitive species indicative of pollutioninduced change in benthic communities. I. Comparative methodology. - Mar. Ecol. Prog. Ser. 9, 111-119.

Hagmeier, A., 1925. Vorläufiger Bericht über die vorbereitenden Untersuchungen der Bodenfauna der Deutschen Bucht mit dem Petersen-Bodengreifer. - Ber. dt. wiss. Kommn Meeresforsch. 1, 247-272.

Hagmeier, A. \& Künne, C., 1951. Die Nahrung der Meeresfische. - Handb. Seefisch. Nordeur. 1, 88-242.

Hertling, H., 1928. Untersuchungen über die Ernährung von Meeresfischen. I. Quantitative Nahrungsuntersuchungen an Pleuronectiden und einigen anderen Fischen der Ostsee. - Ber. dt. wiss. Kommn Meeresforsch. 4, 23-124.

Hulberg, L. W. \& Oliver, J. S., 1980. Caging manipulations in marine soft-bottom communities: importance of animal interactions or sedimentary habitat modifications. - Can. J. Fish. aquat. Sci. 37, 1130-1139.

Höpner-Petersen, G. H., 1977. The density, biomass and origin of the bivalves of the central North Sea. - Meddr Danm. Fisk. - og Havunders. 7, 221-273.

Jenkinson, I. R., 1972. Sludge dumping and benthic communities. - Mar. Pollut. Bull. 3, $102-105$.

Johansen, A. C., 1928. The board of agriculture. - Rep. Dan. biol. Stn 34, 35-51.

Jones, N. S., 1952. The bottom fauna and the food of flatfish off the Cumberland coast. - J. Anim. Ecol. 25, 217-252.

Jumars, P. A., 1975. Environmental grain and polychaete species diversity in a bathyal benthic community. - Mar. Biol. 30, 253-266.

Kühlmorgen-Hille, G., 1963. Quantitative Untersuchungen der Bodenfauna in der Kieler Bucht und ihre jahreszeitlichen Veränderungen. - Kieler Meeresforsch. 19, 42-66.

Lambshead, P. J. D. \& Platt, H. M., 1985. Structural patterns in marine benthic assemblages and their relationship with empirical-statistical methods. In: Proceeding of the 19th European Marine Biology Symposium. Ed. by P. E. Gibbs. Cambridge Univ. Press. Cambridge, 371-380.

Lie, U., 1968. A quantitative study of benthic infauna in Puget Sound, Washington, USA, in 1963-1964. - Fisk. Dir. Skr. (Ser. Havunders.) 14, 229-556.

Lie, U. \& Kisker, D. S., 1970. Species composition and structure of benthic infauna communities off the coast of Washington. - J. Fish. Res. Bd Can. 27, 2273-2287.

Maurer, D., Watling, L., Kinner, P., Leathem, W. \& Wethe, C., 1978. Benthic invertebrate assemblages of Delaware Bay. - Mar. Biol. 45, 65-78.

Maurer, D. \& Aprill, G., 1979. Intertidal benthic invertebrates and sediment stability at the mouth of Delaware Bay. - Int. Revue, ges. Hydrobiol. 64, 379-403.

McCall, P., 1977. Community patterns and adaptive strategies of the infaunal benthos of Long Island Sound. - J. mar. Res. 35, 221-266.

McIntyre, A. D., 1978. The benthos of the Western North Sea. - Rapp. P.-v. Réun. Cons. int. Explor. Mer $172,405-417$.

Mirza, F. B. \& Gray, J. S., 1981. The fauna of benthic sediments from the organically enriched Oslofjord, Norway, - J. exp. mar. Biol. Ecol. 54, 181-207.

Moore, D. C., 1979. A baseline study of the physico-chemical and benthic characteristics of two sewage sludge dumping grounds outside the Firth of Forth, Scotland. - C. M./ICES, E 67.

Mühlenhardt-Siegel, U., 198.5. Die Weichbodengemeinschaft vor der Elbemündung unter dem Einfluß der Klärschlammverklappung. Eine Analyse über Sedimentstruktur, Biomasse, Populationsdynamik. Diss. Univ. Hamburg, $198 \mathrm{pp}$.

Mühlenhardt-Siegel, U., 1981. Die Biomasse mariner Makrobenthos-Gesellschaften im Einflußbereich der Klärschlammverklappung vor der Elbemündung. - Helgoländer Meeresunters. 34. $427-437$.

Mühlenhardt-Siegel, U., Dörjes, J. \& Cosel, R von, 1983. Die amerikanische Schwertmuschel Ensis directus (Conrad) in der Deutschen Bucht. II. Populationsdynamik. - Senckenberg. marit. 15, 93-110.

Müller, G, 1964. Methoden der Sedimentanalyse. Schweizerbart, Stuttgart, 303 pp.

Nichols, $F, H_{1}, 1974$. Sediment tumover by a deposit-feeding polychaete 1974, - Limnol. Oceanogr. 19, 945-950. 
Parker, J. G., 1980. Effects of pollution upon the benthos of Belfast Lough. - Mar. Pollut. Bull. 11, 80-83.

Pearce, J. B., 1972. The effects of solid waste disposal on benthic communities in the New York Bight. In: Marine pollution and sea life. Ed. by M. Ruivo. Fishing News, London, 404-411.

Pearson, T. H., 1971. The benthic ecology of Loch Linnhe and Loch Eil, a sea-loch system on the west coast of Scotland. III. The effects on the benthic fauna of the introduction of a pulp mill effluent. J. exp. mar. Biol. Ecol. 6, 211-233.

Pearson, T. H., 1975. The benthic ecology of Loch Linnhe and Loch Eil, a sea-loch system on the west coast of Scotland. IV. Changes in the benthic fauna attributable to organic enrichment. - J. exp. mar. Biol. Ecol. 20, 1-41.

Pearson, T. H., 1980. Marine pollution effects of pulp and paper industry wastes. - Helgoländer Meeresunters. 33, 340-365.

Pearson, T. H., 1981. Streß and catastrophe in marine benthic ecosystems. In: Streß effects on natural ecosystems. Ed. by G. W. Barrett \& R. Rosenberg. Wiley, New York, 201-214.

Pearson, T. H., 1982. Ecological principles and biochemical indices. - Publs scient. tech. CNEXO (Actualités de Biochemie Marine) 5, 423-434.

Pearson, T. H. \& Rosenberg, R., 1976. A comparative study of the effects on marine environment of wastes from cellulose industries in Scotland and Sweden. - Ambio 5, 77-79.

Pearson, T. H. \& Rosenberg, R., 1978. Macrobenthic succession in relation to organic enrichment and pollution of the marine environment. - Oceanogr. mar. Biol. 16, 229-311.

Pearson, T. H., Duncan, G. \& Nuttall, J., 1982. The Loch Eil project: population fluctuations in the macrobenthos. - J. exp. mar. Biol. Ecol. 56, 305-321.

Pearson, T. H., Gray, J. S. \& Johannessen, P. J., 1983. Objective selection of sensitive species indicative of pollution induced changes in benthic communities. 2. Data analyses. - Mar. Ecol. Progr. Ser. 12, 237-255.

Persson, L. E., 1978. Spatial and temporal variations of juvenile macrofauna on a down-shore transect in Hanoe Bight (Southern Baltic). - Kieler Meeresforsch. (Sonderh.) 4, 103-107.

Pielou, E. C., 1976. Population and community ecology. Gordon \& Breach, New York, $424 \mathrm{pp}$

Probert, P. K., 1981. Changes in the benthic community of china clay waste deposits in Mevagissey Bay following a reduction of discharges. - J. mar. biol. Asss. U. K. 61, 789-804.

Rachor, E., 1977. Faunenverarmung in einem Schlickgebiet in der Nähe Helgolands. - Helgoländer wiss. Meeresunters. 30, 633-651.

Rachor, E., 1980. The inner German Bight - an ecologically sensitive area as indicated by the bottom fauna. - Helgoländer Meeresunters. 33, 522-530.

Rachor, E., 1982. Indikatorarten für Umweltbelastungen im Meer. - Decheniana (Beih.) 26, 128-137.

Rapport, D. J., Regier, H. A. \& Thorpe, C., 1981. Dynamics, prognosis and treatment of ecosystems under stress. In: Stress effects on natural ecosystems. Ed. by G. W. Barrett \& R. Rosenberg. Wiley, New York, 269-280.

Reish, D. J., 1980. Effects of domestic wastes on the benthic marine communities of southern California. - Helgoländer Meeresunters. 33, 377-383.

Rhoads, D. C., 1974. Organism-sediment relation on the muddy sea floor. - Oceanogr. mar. Biol. 12, 263-300.

Rosenberg, R., 1971. Recovery of the littoral fauna in Saltkällefjord subsequent to discontinued operations of a sulphite pulp mill. - Thalassia jugosl. 7, 341-351.

Rosenberg, R., 1974. Spatial dispersion of an estuarine benthic faunal community, - J. exp. mar. Biol. Ecol. 15, 69-80.

Rosenberg, R., 1977. Benthic macrofauna dynamics, production and dispersion in an oxygendeficient estuary of West Sweden. - J. exp. mar. Biol. Ecol. 26, 107-133.

Rosenberg, R., 1980. Effect of oxygen deficiency on benthic macrofauna. In: Fjord oceanography. Ed. by. H. J. Freeland, D. M. Fanner \& C. D. Levings. Plenum Press, New York, 499-514.

Rosenberg, R., Nilsson, K. \& Landner, L., 1975. Effects of a sulphate pulp mill on the benthic macrofauna in a firth of the Bothnian Sea. - Merentutkimuslait. Julk. 239, 289-300.

Sachs, L., 1984. Angewandte Statistik. Springer, Berlin, 552 pp.

Salzwedel, H., Rachor, E. \& Gerdes, D., 1985. Benthic macrofauna communities in the German Bight. - Veröff. Inst. Meeresforsch. Bremerh. 20, 199-267. 
Shaw, K. M., Lambshead, P. J. D. \& Platt,.H. M., 1983. Detection of polluted induced disturbance in marine benthic assemblages with special reference to nematodes. - Mar. Ecol. Prog. Ser. 11, 195-202.

Soulsby, P. G., Lowthian, D. \& Houston, M., 1978. Observations on the effects of sewage discharged into a tidal harbour. - Mar. Pollut. Bull. 9, 242-245.

Steimle, E. W. jr., 1982. The benthic macroinvertebrates of the Block Island Sound. - Estuar. coast. Shelf Sci. 15, 1-16.

Stirn, J., 1973. Organic pollution as the main factor causing biological disequilibria in coastal waters. - Archo Oceanogr. Limnol. 18, 111-119.

Stripp, K., 1969. Jahreszeitliche Fluktuationen von Makrofauna und Meiofauna in der Helgoländer Bucht. - Veröff. Inst. Meeresforsch. Bremerh. 12, 65-94.

Topping, G. \& McIntyre, A. D., 1972. Benthic observations on a sewage sludge dumping ground. C.M./ICES, E 30.

Tulkki, P., 1968. Effects of pollution on the benthos off Gothenburg. - Helgoländer wiss. Meeresunters. 17, 209-215.

Tulkki, P., 1969. Die Bodentiere als Indikatororganismen für Verunreinigungen eines Brackwassergebietes. - Limnologica 7, 79-85.

Tyler, P. A., 1977. Seasonal variation and ecology of gametogenesis in the genus Ophiura (Ophiuroidea: Echinodermata) from the Bristol Channel. - J. exp. mar. Biol. Ecol. 30, 185-197.

Vanosmael, C., Willems, K. A., Claeys, D., Vincx, M. \& Heip, C., 1982. Macrobenthos of a sublittoral sand bank in the southern bight of the North Sea. - J. mar. biol. Ass. U. K. 62, 521-534.

Wikander, P. B., 1981. Quantitative aspects of deposit feeding in Abra nitida (Müller) and $A$. longicallus (Scacchi) (Bivalvia: Tellinacea). - Sarsia 66, 35-48.

Young, D. K. \& Pearce, J. B., 1975. Shell disease in crabs and lobsters from New York Bight. - Mar. Pollut. Bull. 6, 101-105.

Young, D. K. \& Rhoads, D. C., 1971. Animal-sediment relation in Cape Cod Bay, Mass. I. A transect study. - Mar. Biol. 11, 242-254.

Ziegelmeier, E., 1964. Einwirkung des kalten Winters 1962/63 auf das Makrobenthos im Ostteil der Deutschen Bucht. - Helgoländer wiss. Meeresunters. 10, 276-282.

Ziegelmeier, E., 1970. Über das Massenvorkommen verschiedener makrobenthaler Wirbelloser während der Wiederbesiedlungsphase nach Schädigungen durch "katastrophale" Umwelteinflüsse. - Helgoländer wiss. Meeresunters. 21, 9-20.

Ziegelmeier, E., 1978. Macrobenthos investigations in the eastern part of the German Bight from 1950-1974. - Rapp. P.-v. Réun. Cons. int. Explor. Mer 172, 432-444. 